Article

\title{
Evaluation of Combined Use of Drought Indices in the Case of Konya Closed Basin
}

\author{
Huseyin Yildirim Dalkilic* \\ 1 Assistant Professor, Department of Civil Engineering, Erzincan Binali Yildirim University, Erzincan 24000, \\ Turkey, E-mail: hydalkilic@erzincan.edu.tr \\ *Correspondence: hydalkilic@erzincan.edu.tr; Tel.: +90 446 2240088+ 43098.
}

\begin{abstract}
The climate covers a series of events that deeply affect human life. It is possible to understand these events through spatial and statistical analyzes. Today, climate change, which is one of the most important of these events and the impact factors of consequences of this change, become a current issue. Drought is cited as one of the consequences of climate change and it is important to examine it with various methods as it can give negative results to both the economy and the nature. In this study, the drought status of the regions where these stations are located and the effects of drought on climate change were statistically calculated and evaluated using Standardized Precipitation Index (SPI), Percentage of Normal Index (PNI), Aridity Index (AI) and Standardized Precipitation -Evopotranspiration Index (SPEI). The precipitation data from 1981 to 2010 were obtained from Cihanbeyli, Karapınar, Çumra, Seydişehir, Kulu, Ereğli, Niğde, Karaman, Beyşehir and Aksaray meteorology stations affiliated to Turkish State Meteorological Service. At the same time, factor analysis and validity-reliability analysis were conducted to test the computability of the indices used in the study as a single index and to determine the reliability of the operations. While using exploratory factor analysis, Kaiser-Meyer-Olkin (KMO) test and Barlett test for factor analysis; Cronbach's alpha coefficient was used for reliability analysis. In our study, K-Means Cluster Analysis method was performed to determine the cutoff values of indices. According to the result of cluster analysis for the new (common) index, new clusters were created and ANOVA test was conducted to determine whether there was a difference between clusters.
\end{abstract}

Keywords: Aridity Index (AI), Percentage of Normal Index (PNI), Standardized Precipitation Evopotranspiration Index (SPEI), Standardized Precipitation Index (SPI), Drought, Factor Analysis, Reliability Analysis, Cluster Analysis, ANOVA

\section{Introduction}

Natural disasters are events that cause loss of life and property of people and living things. In recent years, large investments have been made to protect against natural disasters and take precautionary pre-disaster measures worldwide. The majority of natural disasters are meteorological disasters, their types and importance sequences are addressed in different ways in different regions. However, drought is one of the most common natural disasters affecting living creatures worldwide. Drought is a very slow-moving and time-consuming disaster, for this reason needs to be carefully examined and monitored. This research and monitoring process is important in order to make out any damages that may occur [1]. Basically, drought is classified as meteorological, agricultural and hydrological and analysis can be done by different methods. Considering the studies carried out by these methods; Sruthi \&Aslam analyzed vegetation stress in their studies using the Normalized Difference Vegetation Index (NDVI) method [2]. In Leng et al.'s study, SPI, SRI (standardized runoff index) and SSWI (standardized soilmoisture index) drought indices were used, by analysing data 
from 1971 to 2000 and the drought condition, drought severity, drought frequency and drought status were estimated from 2020 to 2049 using GCMs (global circulationmodels) [3]. Lee et al. conducted a two-variable drought analysis based on two-component functions. Different marginal distributions for drought duration and severity data for four Canadian and Iranian stations were examined. In this study, it was argued that the relationship between Clayton Copula's drought duration and severity could not be modeled, hence the importance of developing a regional bivariate drought frequency analysis model was suggested [4]. Zelenhasic analyzed the case where the probability of occurence in extreme drought status and extreme drought duration are not equal [5]. Raziei et al., stated that the lack of reliable and updated precipitation datasets in Iran limited setting up an early warning system, and drought analysis was carried out by using SPI method to overcome this obstacle by using GPCC and NCEP / NCAR precipitation datasets. The GPCC dataset is stated to be a useful tool [6]. In the study of Yildırım monthly and annual precipitation values were analyzed statistically using RAINBOW software in Manisa-Salihli, and the expected reliable precipitation values in months and years were evaluated as rainy, normal and arid [7]. Akçay et al., used the same software, monthly and annual reliable precipitation values were determined using the precipitation data of Aydin region [8]. Hınıs, In this study, "Normalized Precipitation Index" (NPI) method and "Integrated Drought Index" (IDI) method were compared for the drought analysis of Aksaray province. As a result of this comparison, it was observed that the transitions between wet and dry periods were calculated more frequently and the wetlands were more severe in the integrated drought index [9]. Gümüs et al., the Current Drought Index (CDI) method was used to determine the hydrological drought using monthly current data and temporal drought values were calculated by taking into account the time scales of 1, 3, 6 and 12 month [10]. In the study of Huang et al., SPI and (EDI) (EffectiveDrought Index) were used to determine the drought condition and IPCC Fifth Assessment report (AR5) and RCP 8.5 (RepresentativeConcentrationPathway) scenario were utilised to estimate the regional precipitation and temperature data [11].

In addition to these methods, the studies conducted with the indices of SPI, SPEI, AI and PNI, which are also used in our study; In the studies of Mehr et al., drought analysis was conducted using the regionally adjusted outputs using three meteorological global climate models (GCMs). In the study where SPI and SPEI drought indices were used for drought assessment, the RCP 4.5 and RCP 8.5 scenarios were also used to estimate the near future status of the drought [12]. AKBARI et al., the drought status in the semi-arid regions was analyzed using the SPI and RDI indices and the situation was depicted by simulations created with the help of GIS. In addition to that, with the help of data from 1984 to 2010, using the HadCM3, IPCM and GFC models, evapotranspiration, precipitation house maximum-minimum precipitation values were modeled from 2011 to 2030 [13]. Mishra \& Singh, drought was evaluated hydrologically and climatologically and the effects of drought on the world were examined. It was also stated in the study that paleoclimatological drought calculations included effective drought determination methods. It is also stated that the assessment of drought, both hydrologically and climatologically and paleoclimatologically, gains more significance with climate scenarios [14]. Labedzki, SPI method was used to estimate the frequency of meteorological drought, and the SPI timeline was specified as the best method to show agricultural and hydrological drought under climatic conditions [15]. Hayes et al., drought was investigated using SPI, and it was argued that drought dynamics varied by regions, although drought was across the region [16]. Tsakiris et al., Reconnaissance Drought Index (RDI) and SPI were used [17]. Fiorillo \& Guadagno, based on long hydrological time series, the correlation between karst spring discharge series and precipitation was analyzed using the SPI method and focused on drought periods in the analysis [18]. PAMUK et al., drought occurrences were analyzed by SPI method[19]. Çelik et al., in the study aiming to reveal the drought trend, SPI method was used. In addition to these analyzes, according to Thornthwaite, Aydeniz De Martonne and Erinç, climate classifications were made and long-term annual seasonal drought status in the region was demonstrated [20]. Ilgar, 12-month (annual) and 3month (seasonal) drought analyzes were performed for Çanakkale with the SPI method [21]. VicenteSerrano et al., SPEI method was used in the study and the effects of temperature variability on drought assessment were determined. However, according to the most common drought indices 
using PET values, it was emphasized that the identification of different drought types and effects in the context of global warming of SPEI's multiskalar properties is a significant advantage for SPEI [22]. Nastos et al., spatial and temporal variability of drought was investigated using AI [23]. Greve et al., drought assessment was made using AI, it was also stated that it is more accurate to analyze model outputs rather than using climate models directly [24]. Dave et al., AI was used to monitor desertification status [25]. Nikbakht et al., PNI method was used for drought analysis [26]. Li et al., in order to evaluate spatial and temporal variations of water quality in the Lower Lakes in Australia, statistical methods such as cluster analysis (CA), discriminant analysis (DA), principal component analysis (PCA) and factor analysis (FA) were used [27]. Hasaninenajah et al., In their study on drought effects and drought risk management, statistical analysis was done by SPSS program which was used to determine the reliability level of Cronbach's alpha coefficient, a safety rate of $67 \%$ was obtained [28]. Pham et al., the Effective Drought Index (EDI) was used to determine drought, and statistical analysis was performed using the Barlett-Lewis model [29]. Beharry et al., the SPI method was used to determine the drought situation of the region and KMO (Kaiser - Meyer - Olkin) and Barlett test, prior to principal component analysis, was utilised to check the suitability of data for factor analysis. Factor analysis was also conducted in the study [30]. Devisti \& Motamed, in order to investigate the socio-economic and environmental effects of drought, Cronbach's alpha coefficient was used to determine the level of reliability [31].Albert et al., In the study on the diversity of climate functions, exploratory factor analysis was used to estimate the distribution of climate functions [32]. Biamah et al. aimed to simulate the behaviour of dry and wet precipitation in the Iiuni basin in Kenya stochastically. It is concluded that agricultural drought periods with varying severity and duration occur in Kenya, which has a semi-arid climate. The Iiuni basin precipitation data were used for the variance analysis (ANOVA) and it was revealed that the annual and seasonal precipitation variability between the stations in the Iiuni basin was not significant and therefore the daily precipitation data of the liuni station had been used to show the precipitation characteristics of the entire basin in this region [33]. . In the study conducted by Rahmat et al., the region was divided into homogeneous groups and estimations were made on drought risks. Drought frequency was analyzed in the regions separated by using cluster analysis and Modified Andrews curve, and SPI was used to determine the drought [34]. Borgonovo et al. searched for methods to obtain a better understanding of hydrological model studies conducted for uncertainty measurement and model differentiation in the study. One-way ANOVA sensitivity functions were used for trend analysis [35].

\section{Materials and Methods}

\subsection{Standardized Precipitation Index (SPI)}

The Standard Precipitation Index (SPI), developed by McKee et al. in 1993 to monitor drought, is one of the most common methods in the research of drought in the world. The strongest aspect of SPI is its ability to be calculated for various time scales. This feature enables SPI to monitor shortterm water resources such as soil moisture, which is important for agricultural production, and longterm water resources such as groundwater sources, stream and lake and reservoir levels [14]. The Standardized Precipitation Index (SPI) for any location is calculated with long-term precipitation data for a desired period. On the basis of SPI, it is obtained by dividing the difference of precipitation from the mean of precipitation within a specified time period by the standard deviation $[36,37]$.

SPI: Standard Precipitation Index,

$\mathrm{X}$ : Current precipitation,

$\mathrm{Xi}$ : Average precipitation,

бi: Standard deviation value, SPI can be formulated as follows:

$S P I=\left(X-X_{i}\right) / \sigma_{i}$

As a result of this calculation, drought can be classified according to the classification ranges in Table 1. by using the obtained values. In this assessment; the period when the index values are 
constantly negative is defined as the dry period, while the first value where the index falls below zero is considered as the starting point of the drought. When the index rises to positive value, the end of the drought period is symbolized. [38]. The classification was made according to the SPI method was set out in Table 1.

Table 1. . Drought Classification According to SPI Method [36].

\begin{tabular}{ll}
\hline SPI INDEX VALUES & CLASSIFICATION \\
\hline$\geq 2,0$ & EXTRAORDINARY DAMP (EO.D.) \\
$1,60-1,99$ & SEVERE DAMP (S.D.) \\
$1,30-1,59$ & VERY DAMP (V.D.) \\
$0,80-1,29$ & MIDDLE DAMP (M.D.) \\
$0,51-0,79$ & SEMI (LIGHT) DAMP (S.D.) \\
$0,50-(-0,50)$ & NORMAL (N) \\
$-0,51-(-0,79)$ & SEMI (LIGHT) ARID (S.A.) \\
$-0,80-(-1,29)$ & MIDDLE ARID (M.A.) \\
$-1,30-(-1,59)$ & VERY ARID (V.A.) \\
$-1,60-(-1,99)$ & SEVERE ARID (SV.A.) \\
$\leq-2,0$ & EXTRAORDINARY ARID (EO.A.) \\
\hline
\end{tabular}

\subsection{Percent of Normal Index (PNI)}

Among the drought indices, the easiest to calculate index was considered as PNI. It was obtained by dividing the amount of precipitation to the average of precipitation within a specified time period. In PNI calculations, it is possible to calculate precipitation in annual or monthly periods [39].

PNI: Percent of Normal Index

P: Current precipitation

Pi (Ort.): Then PNI can be calculated with this formula;

$P N I=\left(P_{i} / P_{i(o r t)}\right) \times 100$

According to the results of drought calculation using PNI method, classification can be made as stated in Table 2. on the basis of percentage rate .

Table 2. Drought Classification According to PNI Method [39].

\begin{tabular}{ll}
\hline PNI INDEX VALUES & CLASSIFICATION \\
\hline PNI $\% 75$ & NORMAL (N) \\
$\% 65-\% 75$ & SEMI (LIGHT) ARID (S.A.) \\
$\% 55-\% 65$ & MIDDLE ARID (M.A.) \\
PNI $\% 55$ & SEVERE ARID (SV.A.) \\
\hline
\end{tabular}

\subsection{Standardized Precipitation-Evapotranspiration Index (SPEI)}

The SPEI drought calculation method is based on the potential evapotranspiration (PET) balance, which is the monthly climate balance. Temperature and precipitation data are used in its calculation. The SPEI method can be calculated over multiple time periods [40, 41]. The classification made according to the SPEI method is set out in Table 3. 
Table 3. Drought Classification Made According to SPEI Method [40, 42].

\begin{tabular}{ll}
\hline SPEI & SINIFLANDIRMA \\
\hline$\leq 2,0$ & EXTREME DAMP(EX.D.) \\
$1,5-1,99$ & VERY DAMP(V.D.) \\
$1,0-1,49$ & MIDDLE DAMP(M.D.) \\
$0,0-0,99$ & SEMI (LIGHT) DAMP(S.D.) \\
$0,0-(-0,99)$ & SEMI (LIGHT) ARID (S.A.) \\
$(-1,0)-(-1,49)$ & MIDDLE ARID (M.A.) \\
$(-1,5)-(-1,99)$ & SEVERE ARID (SV.A.) \\
$-2,0 \geq$ & EXTREME ARID (EX.A.) \\
\hline
\end{tabular}

The following methods are used to calculate SPEI;

The following methods are used to calculate SPEI; To calculate the monthly evapotranspiration, if the average temperature value for each month is symbolized by $(\mathrm{T})$;

$i=\left(\frac{T}{5}\right)^{1,514}$

The calculation is made for 12 months of each year and the results are summed. The total value is defined as the annual temperature index (I).

The coefficient $\mathrm{A}$ is calculated using the annual temperature coefficient (Eq. (4)).

$A=6,75 \times 10^{-7} \times I^{3}-7,71 \times 10^{-5} \times I^{2}+1,79 \times 10^{-2} \times I+0,492$

Potential evapotranspiration (PET) value for annual temperature index (I) (Eq. (5));

$P E T=16 \times K\left(\frac{10 \times T_{m m}}{I}\right)^{m}$

The corrected potential evapotranspiration value is calculated by multiplying the potential evapotranspiration (PET) value and the lLatitude Adjustment Coefficient (LAC) value that varies according to the latitude of the region where the drought analysis is performed.

$D E P O T=P E T \times E D K$

Subtracting DEPOT from the monthly total precipitation value of each month, the value of Di is found (Eq. (6)). The study time range is sorted from small to large and given the sequence number. The value of 0.35 is subtracted from each row number, divided by the total number of observations and this value is defined as Fi. $(I=1,2,3$, n)

$F i=(i-0,35) / n$

Each Fi value is calculated as (1-Fi) and then the 0th force of the (1-Fi) value is taken and multiplied by the Di value. This is done for all observations, then the new array is averaged and the result is assigned as $\mathrm{w} 0$. The exponent of the instant operation (1-Fi) value is taken as 1 and 2, respectively, and $\mathrm{w} 1$ and $\mathrm{w} 2$ values are also assigned. Then the coefficients are calculated with the following formulas; 


$$
\begin{gathered}
\beta=\frac{2 \times w_{1}-w_{0}}{6 \times w_{1}-w_{0}-6 \times w_{2}} \\
\alpha=\frac{\left(w_{0}-2 \times w_{1}\right) \times \beta}{\Gamma\left(1+\frac{1}{\beta}\right) \Gamma\left(1-\frac{1}{\beta}\right)}
\end{gathered}
$$

Here, Gamma Function calculation is made $\Gamma(1+1 / \beta)$ or $\Gamma(1-1 / \beta)$ if the value is between 1 and 2 , the gamma value is found from the table. If the value is between 0 and 1 , it is calculated $\Gamma(\alpha)=\Gamma(\alpha+1) / \alpha$ is calculated and $\Gamma(\alpha+1)$ found in the table and divided by $\alpha$ (Eq. (10)).

$F(x)=\left[1+\left(\frac{\alpha}{x-y}\right)^{\beta}\right]^{-1} ; \mathrm{x}=$ values in original order

If $P=1-F(x)$; if $P>0,5$ then P replaces with $1-P$ and the sign of SPEI is reversed (Eq. (1-13)) (Here the last SPEI value is multiplied by (-1)).

$W=\sqrt{-2 \times \ln (P)} ;$ for $P \leq 0,5$

$W=\sqrt{-2 \times \ln (1-P)} ;$ for $\quad P>0,5$

$S P E I=\frac{c_{0}+c_{1} \times w+c_{2} \times w^{2}}{1+d_{1} \times w+d_{2} \times w^{2}+d_{3} \times w^{3}}$

$\begin{array}{lll}\mathrm{C} 0=2,515517 & ; & \mathrm{d} 1=1,432788 \\ \mathrm{C} 1=0,802853 & ; & \mathrm{d} 2=0,189269 \\ \mathrm{C} 2=0,010,28 & ; & \mathrm{d} 3=0,001308\end{array}$

\subsection{Aridity Index (AI)}

Aridity precipitation index is defined as the continuous climatological drought caused by the long-term atmosphere circulation existing in any part of the earth. It is calculated by dividing the precipitation that occurs in certain periods by the potential PET value of that period [36].

$\mathrm{P}$, sum of precipitation $(\mathrm{mm})$,

PET, potential evapotranspiration ( $\mathrm{mm}$ ) then it can be calculated by this formula;

$A I=P / P E T$

Calculated with the Eq. (14).

Classification made according to AI method is set out in Table 4.

Table 4. Classification and scoring of UNEP- UNCCCD Aridity Index (AI) values in terms of desertification assessment [43]. 


\begin{tabular}{lll}
\hline AI & POINT & CLASSIFICATION \\
\hline$>2$ & 1 & EXTREME DAMP (EX.D.) \\
$2-1$ & 1,2 & VERY DAMP AND DAMP (V.D.\&D.) \\
$1-0,80$ & 1,4 & LIGHT DAMP (L.D.) \\
$0,80-0,65$ & 1,6 & SEMI DAMP (DAMP) (S.D. (D.)) \\
$0,65-0,50$ & 1,8 & SEMI DAMP (ARID)(S.D.(A.)) \\
$<0,50$ & 2 & SEMI ARID AND ARID (S.A.\&A.) \\
\hline
\end{tabular}

\subsection{Statistical Calculation Methods}

\subsubsection{Factor Analysis}

Factor analysis is one of the multivariate statistical techniques that make a large number of variables related to each other into fewer meaningful independent factors. The aim of this analysis is to reduce the number of variables and to reveal the structure of the relationship between variables. One of the most widely used types of factor analysis is exploratory factor analysis, which is also used in this study. Exploratory factor analysis is a method of factor analysis that is found to have fewer than related $p$ number variables and is used to derive new factors independently. Exploratory factor analysis is used to discover the data to determine the properties and number of factors that explain the covariance between the variables when a research does not have sufficient information to establish a hypothesis about the factor or variable amount that underlies the data [44]. There are four basic steps in exploratory factor analysis and factor analysis in general.

These steps are;

1. Determination of the appropriateness of the dataset for factor analysis

2. Extraction of factors (Determination of factors)

3. Rotation of the Factors

4. Naming of the factors.

In the first step, the Barlett test or Kaiser-Meyer-Olkin (KMO) can be used to create the correlation matrix to evaluate the suitability of the data set. $\mathrm{KMO}$ is defined as the criterion applied to measure the strength of the relationship between variables. In order to implement satisfactory factor analysis, $\mathrm{KMO}$, the sample must be greater than 0.5. In the case of variables less than 0.5 , these values are not included in the analysis. The Bartlett test is also performed to measure the relationship magnitude between variables such as KMO. However, Barlett is not a criterion, but a test statistic. The sample used in the test must be less than 0.05 [45]. KMO;

$$
K M O=\frac{\sum_{i \neq j} \sum r_{i j}{ }^{2}}{\sum_{i \neq j} \sum r_{i j}{ }^{2}+\sum_{i \neq j} \sum a_{i j}{ }^{2}}
$$

is calculated by (Eq. (15), Barlett is; 


$$
x^{2}=-\left[(N-1)-\left(\frac{2 k+5}{l}\right)\right] \ln |R|
$$

It can be calculated with the formula. Here, the value obtained in the $x^{2}$ Barlett test, $\mathrm{N}$ is the sample size, $\mathrm{k}$ is the number of items, and $|R|$ is the determinant of the correlation matrix obtained from the data.In the second step, Eigenvalues statistics, scree test, percentage of total variance management, Joiliffe criterion, explained variance criterion can be used or the number of factors can be determined by the researcher as in this study. In the third step, rotation of the of the factors, the aim is to obtain nameable and interpretable factors. In the last step, the naming of the factors is made $[30,32,46]$.

\subsubsection{Reliability Analysis}

There are many points to be considered when creating a reliable measurement tool. Therefore, the properties and reliability of the measurements made on the variables and the questionnaires, scales or tests used in the measurement should be evaluated. Models used for reliability analysis; Cronbach's alpha coefficient is split-split model, Guttman model and parallel model. The alpha coefficient of Cronbach used in this study is related with whether the k problem in the scale shows a homogeneous structure or not. The coefficient, which is also expressed as the weighted standard change average, is the average of the total of the variances of the $\mathrm{k}$ problem in the scale, and this coefficient, which can take a value between 0 and 1, is obtained by summing the total score of the unit for each question in the scale. If the correlation between the questions is negative, the coefficient calculated with the Alpha method is also negative. This situation causes the reliability model to deteriorate. Depending on the alpha ( $\alpha$ ) coefficient, the reliability of the scale is set out in Table 5 [47];

Table 5. Reliability classification of Alpha coefficient [47]

\begin{tabular}{ll}
\hline ALFA $(\boldsymbol{\alpha})$ & RELIABILITY \\
\hline $0.00 \leq \alpha \leq 0.40$ & Not safe \\
$0.40 \leq \alpha \leq 0.60$ & Low reliability \\
$0.60 \leq \alpha \leq 0.80$ & pretty reliable \\
$0.80 \leq \alpha \leq 1.00$ & highly reliable \\
\hline
\end{tabular}

\subsubsection{Cluster Analysis}

Cluster analysis is a statistical technique that divides observations into similar sets or groups. This analysis method, which is used to analyze a set of clusters with the aim of finding homogeneous or well separated subsets of these clusters, consists of 3 stages. Data entry is prepared, data is recorded, and then the distance matrix is obtained in the first stage. In the second stage, clustering method is determined and applied, and in the last stage, the results are evaluated [48-50]

It is desired to determine the similarities or distances between individuals or objects observed in cluster analysis. Similarity or distance criteria are used in order to carry out this process. Although 
these criteria are numerous, they are basically chosen based on whether the data is quantitative or mixed. For quantitative data, the calculation is done by using mathematical formulas such as Minkowski distance, Manhattan City-Block distance, Euclidean distance, and Mahalanobis distance. However, it is not always possible for all variables to consist of quantitative data. In this case, the distance between the $\mathrm{x}_{\mathrm{i}}$ and $\mathrm{x}_{\mathrm{j}}$, each containing $P$ number quantitative variables, is:

$d_{i j}=d\left(x_{i}, x_{j}\right)$

$w_{k}=\left(\begin{array}{cl}1 & \begin{array}{ll}1 & \text { (quantitative data) } \\ \text { (qualitative data) }\end{array}\end{array}\right)$

$d\left(x_{i}, x_{j}\right)=\frac{1}{p} \sum_{k=1}^{p} w_{k}\left|x_{i k}-x_{j k}\right|$

Then, it is expressed with the formula below [50]. After determining the distance, the number of clusters must be known in order to make up the results meaningfully. Number of clusters (k) is;

$k=(n / 2)^{1 / 2}$

\subsubsection{One-way Analysis of Variance (ANOVA)}

The variance analysis (ANOVA), which tests whether the difference between the averages of the data in two or more data groups is significant, and also which is very important in discovery-confirmatory data analysis, can be classified according to the status of the data groups and variables. One of the most used variance analysis is the one-way analysis of variance (one way ANOVA) that tests the averages of two or more data groups [51, 52]. ANOVA's main assumptions are as follows:

1. The measurements of the dependent variable are at least in the range scale,

2. The scores show a normal distribution for the factor investigated in the dependent variable,

3. There is no relationship between the samples that meet the average scores,

4. The variances related to the dependent variable are equal for each sample.

$\mathrm{KT} T=$ Total sum of squares,

$\mathrm{KT}_{\mathrm{A}}=$ Sum of intergroup squares caused by factor $\mathrm{A}$,

$K T_{e}=$ If the error symbolizes the sum of squares, the total of the statistical model squares for ANOVA is;

$K T_{T}=K T_{A}+K T_{e}$

$\mathrm{n}=$ total number of subjects,

$\mathrm{A}=$ If the level and the mean of the squares of the factor, the effect of which on the dependent variable is investigated, define the variance, then the formulas for one-way analysis of variance should be as given in Table 6.

Table 6. One-way ANOVA formulas [53] 


\begin{tabular}{|c|c|c|c|c|}
\hline VARIANCF & TOTAL OF & DEGREE OF & AVERAGE OF & \\
\hline SOURCE & $\begin{array}{l}\text { SQUARES } \\
(\mathrm{KT})\end{array}$ & $\begin{array}{l}\text { INDEPENDENCE } \\
\text { (sd) }\end{array}$ & $\begin{array}{l}\text { SQUARES } \\
(\mathrm{KO})\end{array}$ & RATIO OF F \\
\hline INTERGROUP & $\mathrm{KT}_{\mathrm{A}}$ & A-1 & {$\left[\mathrm{KT}_{\mathrm{A}} / \mathrm{A}-1\right]=\mathrm{KO}_{\mathrm{A}}$} & $\mathrm{KO}_{\mathrm{A}} / \mathrm{KO}_{\mathrm{e}}$ \\
\hline IN GROUPS & $\mathrm{KT}$ e & $\mathrm{n}-\mathrm{A}$ & {$[\mathrm{KTe} / \mathrm{n}-\mathrm{A}]=\mathrm{KOe}$} & \\
\hline TOTAL & KT & $\mathrm{n}-1$ & & \\
\hline
\end{tabular}

When ANOVA is applied for two groups and if the difference between the two mean values is found significant, the sizes of the mean values are taken into consider and then the results are interpreted. Various applications such as null hypothesis can be made for three or more samples. Eta-square $\left(n^{2}\right)$, the most commonly used correlation coefficient in determining the strength of the relationship between variables in an ANOVA pattern, is calculated by [53];

$\eta^{2}=K T_{\text {intergroup }} / K T^{\text {total }}$

\section{WORKING AREA AND DATA}

This study was carried out in Konya Closed Basin (KCB), located between the $36^{\circ} 51^{\prime \prime}-39^{\circ} 29^{\prime \prime}$ latitudes and the $31^{\circ} 36^{\prime \prime}-34^{\circ} 52^{\prime \prime}$ longitudes in the Central Anatolia Region/Turkey, with an average altitude of $1000 \mathrm{~m}$. Konya Closed Basin has 5.42648 million ha of surface area to evolve, and this value is approximately $7 \%$ of Turkey's surface. The specified basin, is Turkey's largest closed basin [54]. In this study, meteorological data used for drought analysis, was obtained from Turkish State Meteorological Service. Station names and geographic location information of Konya Closed Basin are set out in Table 7. , and the map of the basin is depicted in Figure 1.

Table 7. Konya Closed Basin Map Display

\begin{tabular}{llllll}
\hline $\begin{array}{l}\text { STATION } \\
\text { NUMBER }\end{array}$ & $\begin{array}{l}\text { STATION } \\
\text { NAME }\end{array}$ & \multirow{2}{*}{ PROVINCE } & DISTRICT & \multirow{2}{*}{ LATITUDE } & \multirow{2}{*}{ LONGITUDE } \\
\hline 17191 & Cihanbeyli & Konya & Cihanbeyli & 38.650578 & 32.921860 \\
17192 & Aksaray & Aksaray & Merkez & 38.3705 & 33.9987 \\
17242 & Beyşehir & Konya & Beyşehir & 37.6777 & 31.7463 \\
17246 & Karaman & Karaman & Merkez & 37.1932 & 33.2202 \\
17248 & Ereğli & Konya & Ereğli & 37.5255 & 34.0485 \\
17250 & Niğde & Niğde & Merkez & 37.9587 & 34.6795 \\
17754 & Kulu & Konya & Kulu & 39.0788 & 33.0657 \\
17898 & Seydişehir & Konya & Seydişehir & 37.4267 & 31.8490 \\
17900 & Çumra & Konya & Çumra & 37.5658 & 32.7900 \\
17902 & Karapınar & Konya & Karapınar & 37.714722 & 33.526111 \\
\hline
\end{tabular}




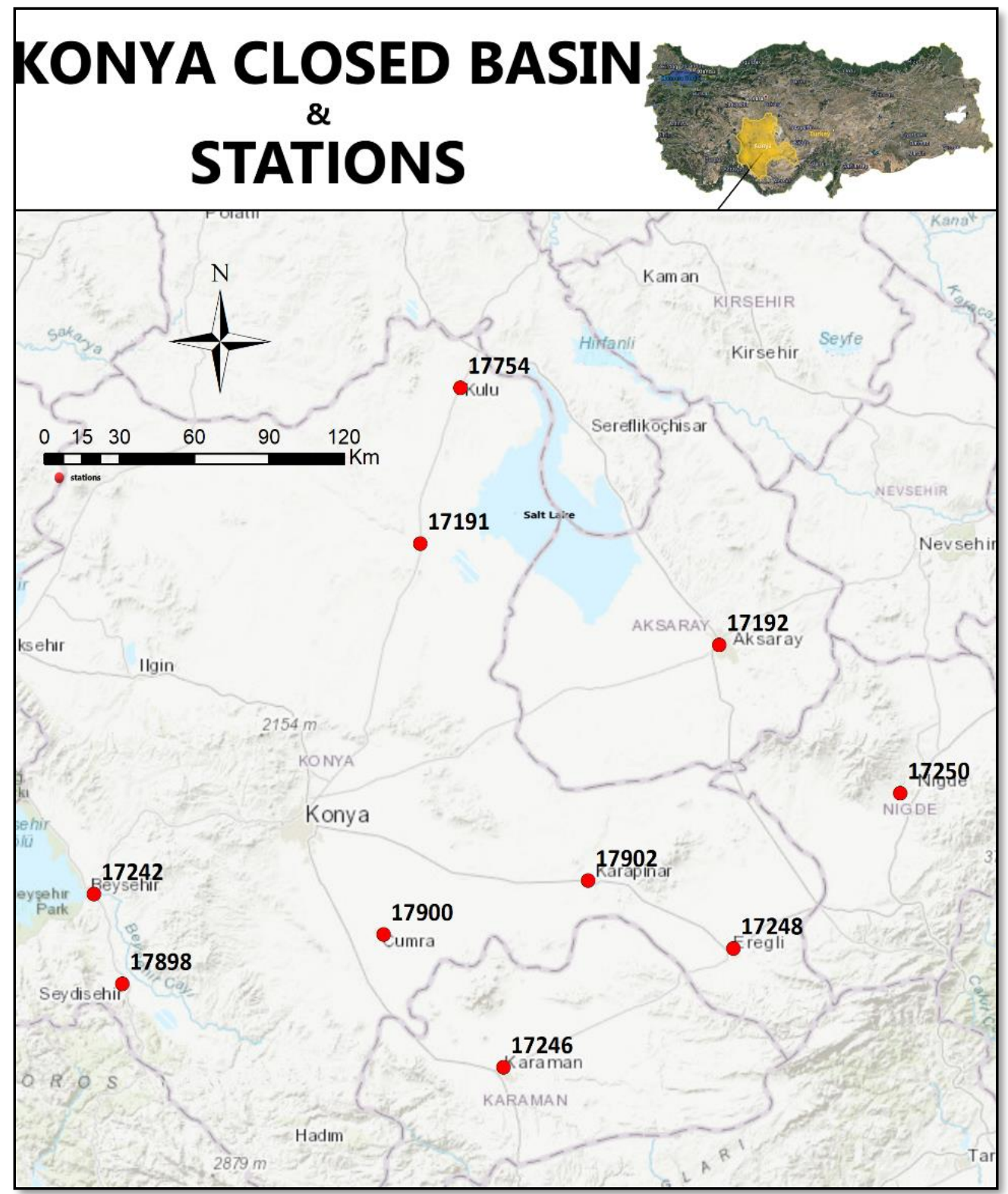

Figure 1. Konya Closed Basin Map Display and Stations [55]. 
Table 8. Cihanbeyli, Aksaray, Beyşehir, Karaman and Ereğli stations index results -1

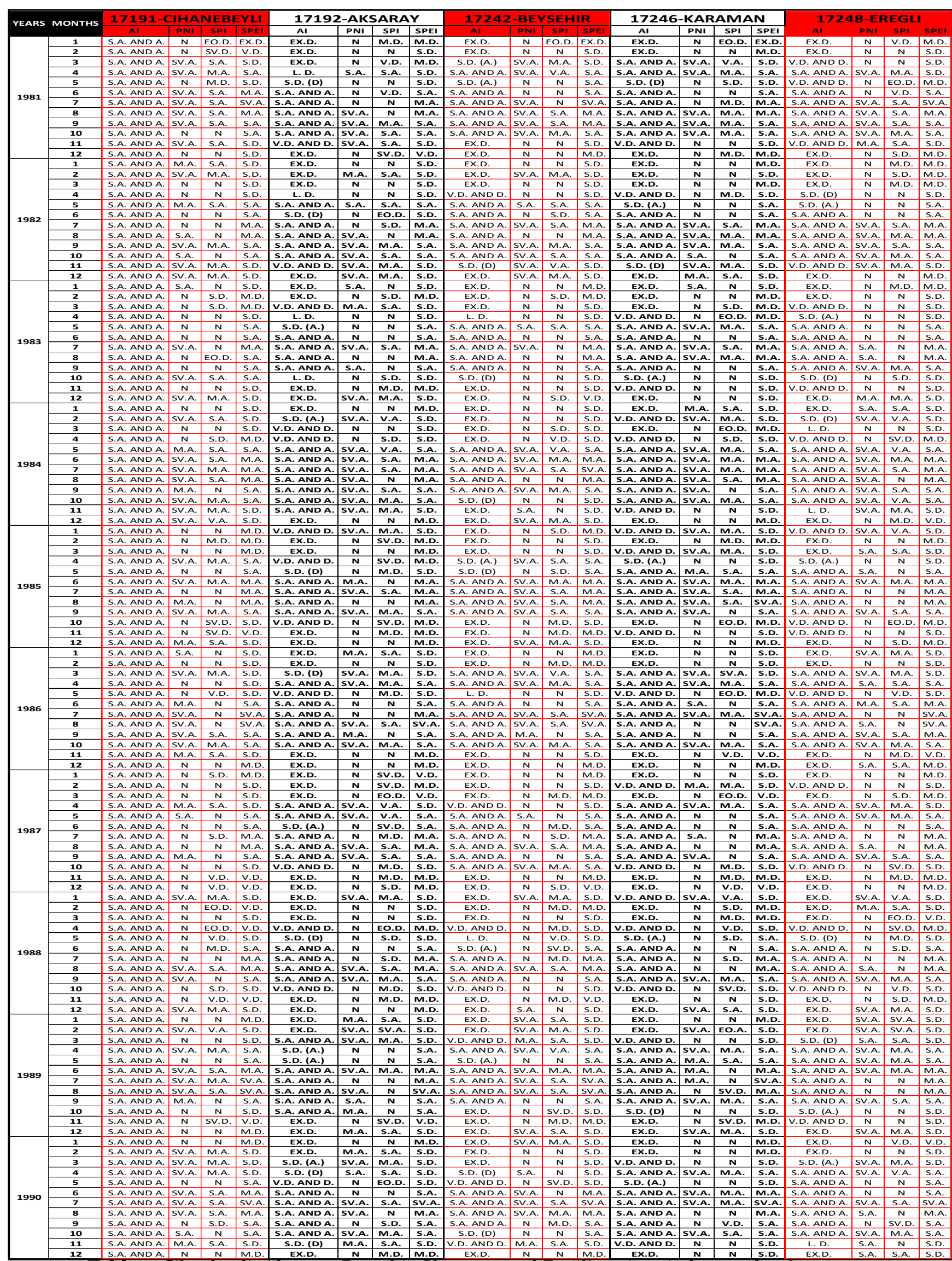

Table 9. Cihanbeyli, Aksaray, Beyşehir, Karaman and Eregli stations index results -2

\begin{tabular}{|c|c|c|c|c|c|c|c|c|c|c|c|c|c|c|c|c|c|c|c|c|c|}
\hline \multirow{2}{*}{ YEARS } & \multirow{2}{*}{ MONTHS } & \multicolumn{4}{|c|}{ 17191-CIHANEBEYLI } & \multicolumn{4}{|c|}{ 17192-AKSARAY } & \multicolumn{4}{|c|}{ 17242-BEYSEHIR } & \multicolumn{4}{|c|}{ 17246-KARAMAN } & \multicolumn{4}{|c|}{ 17248-EREGLI } \\
\hline & & Al & PNI & SPI & SPEI & Al & PNI & SPI & SPEI & Al & PNI & SPI & SPEI & Al & PNI & SPI & SPEI & AI & PNI & SPI & $\mathrm{EI}$ \\
\hline \multirow{9}{*}{195} & $\mathbf{1}$ & S.A. AND A. & S.A. & $\mathrm{N}$ & S.D. & & & & M.D. & EX.D. & $\mathrm{N}$ & $\mathrm{N}$ & S.D. & & & st & & EX.D. & $\mathrm{N}$ & M.D. & M.D. \\
\hline & 2 & S.A.A & $\mathrm{N}$ & $\mathrm{N}$ & M.D. & E) & $\mathbf{N}$ & $\pi$ & M.D. & EX.D. & $\mathrm{N}$ & $\mathbf{N}$ & & & & & & Ex & M.A. & M.A. & \\
\hline & 3 & S.A. AND A. & $\mathrm{N}$ & $\mathrm{N}$ & S.D. & S.D & SV.A. & M.A. & S.D. & S.A. AND A. & SV.A. & V.A. & S.A. & D) & M.A. & S.A. & b. & L. D. & $\mathrm{N}$ & $\mathrm{N}$ & S.D. \\
\hline & 4 & S.A. AND A. & $\mathrm{N}$ & EO.D. & V.D. & V.D.A & $\mathbf{N}$ & S.D. & S.D. & V.D. AND D. & $\mathrm{N}$ & S.D & S.D & S.D. & $\mathbf{N}$ & $\mathbf{N}$ & & L. 5 & $\mathrm{~N}$ & S.D. & D. \\
\hline & 5 & S.A. AND A. & $N$ & $\mathrm{~N}$ & S.D. & S.D & & & & S.A. An & M.A. & s. & & S.A.A & SV.A. & & & S.A. AND A. & S.A. & $\mathrm{N}$ & S.A. \\
\hline & 56 & S.A. An & cye & $N$ & M.A. & S.A.A & SV.A. & M.A. & M.A. & \begin{tabular}{|l|} 
S.A. An \\
\end{tabular} & SV.A. & M.A. & M. & S.A.A & SV.A. & M.A. & M.A. & S.A. A & $N$ & $N$ & S.A. \\
\hline & 7 & S.A. Al & SV.A. & M.A. & SV.A & S.A. AND A. & SV.A. & $\mathbf{N}$ & SV.A. & S.A. An & $N$ & EO.D. & M.A. & S.A. AND A. & SV.A. & M.A. & SV.A. & S.A. A & $\mathrm{N}$ & S.D. & M.A. \\
\hline & 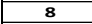 & S.A. AND A. & SV.A. & S.A. & M.A. & S.A. AND A. & SV.A. & $\mathbf{N}$ & SV.A. & S.A. AND A. & SV.A. & S.A. & SV.A. & S.A. AND A. & $\mathbf{N}$ & V.D. & M.A. & S.A. AND A. & $\mathrm{N}$ & $\mathrm{N}$ & M.A. \\
\hline & 9 & S.A. AND A. & SV.A. & M.A. & S.A. & S.A. AND A & SV.A. & S.A. & S.A. & S.A. AND A. & $N$ & $\mathrm{~N}$ & S.A. & S.A.AND A. & SV.A. & S.A. & \begin{tabular}{|l|} 
S.A. \\
\end{tabular} & S.A. AND A. & $N$ & $N$ & S.A. \\
\hline
\end{tabular}


Table 10. Cihanbeyli, Aksaray, Beyşehir, Karaman and Ereğli stations index results -3

\begin{tabular}{|c|c|c|c|c|c|c|c|c|c|c|c|c|c|c|c|c|c|c|c|c|c|}
\hline \multirow{2}{*}{ YEARS } & \multirow{2}{*}{ MONTHS } & \multicolumn{4}{|c|}{ 17191-CIHANEBEYLI } & \multicolumn{4}{|c|}{ 17192-AKSARAY } & \multicolumn{4}{|c|}{ 17242-BEYSEHIR } & \multicolumn{4}{|c|}{ 17246-KARAMAN } & \multicolumn{4}{|c|}{ 17248-EREGLI } \\
\hline & & Al & PNI & SPI & SPEI & Al & PNI & SPI & SPEI & Al & PNI & SPI & SPEI & Al & PNI & SPI & SPEI & & PNI & SPI & SPEI \\
\hline \multirow{10}{*}{2001} & 1 & S.A. AND A. & $\mathrm{N}$ & SV.A. & M.D. & S.A. AND A. & SV.A. & SV.A. & S.D. & S.D. (A.) & SV.A. & V.A. & S.D. & S.A. AND A. & SV.A. & SV.A. & S.D. & AND A & SV.A. & EO.A. & \\
\hline & 2 & S.A. AND A. & $\mathrm{N}$ & $\mathrm{N}$ & M.D. & & $\mathbf{N}$ & $\mathbf{N}$ & S.D. & EX.D. & $\mathrm{N}$ & $\mathrm{N}$ & S.D. & EX.D. & $\mathbf{N}$ & $\mathbf{N}$ & S.D. & EX.D. & $\mathrm{N}$ & M.D. & M.D. \\
\hline & 3 & S.A. AND A. & SV.A. & M.A. & S.A. & S.D. (A.) & M.A. & S.A. & S.A. & S.D. (D) & M.A. & S.A. & S.D. & S.D. (D) & $\mathbf{N}$ & $\mathbf{N}$ & S.D. & S.D. (D) & $\mathrm{N}$ & $\mathrm{N}$ & S.D. \\
\hline & 4 & S.A. AND A. & SV.A. & M.A. & S.A. & S.D. (A.) & M.A. & M.A. & \begin{tabular}{|l|} 
S.A. \\
\end{tabular} & V.D. AND D. & $\mathrm{N}$ & $\mathrm{N}$ & S.D. & S.A. AND A. & S.A. & S.A. & S.A. & S.A. AND A. & M.A. & S.A. & S.A. \\
\hline & 6 & S.A. AND A. & SV.A. & M.A. & M.A. & S.A. AND A. & SV.A. & M.A. & M.A. & S.A. AND A. & SV.A. & M.A. & M.A. & & $\mathbf{N}$ & EO.D. & S.A. & S.A. AND A. & $N$ & $\mathrm{~N}$ & M.A. \\
\hline & 7 & S.A. AND A. & SV.A. & M.A. & SV.A. & \begin{tabular}{|l|} 
S.A. AND A. \\
\end{tabular} & SV.A. & S.A. & SV.A. & S.A. AND A. & S.A. & $\mathrm{N}$ & SV.A. & S.A. AND A. & $N$ & $\mathbf{N}$ & SV.A. & S.A. AND A. & SV.A. & S.A. & SV.A. \\
\hline & 8 & S.A. AND A. & SV.A. & S.A. & SV.A. & S.A. AND A. & SV.A. & S.A. & SV.A. & S.A. AND A. & $\mathrm{N}$ & $\mathrm{N}$ & M.A. & S.A. AND A. & SV.A. & S.A. & SV.A. & S.A. AND A. & SV.A. & M.A. & SV.A. \\
\hline & 9 & S.A. AND A. & SV.A. & S.A. & M.A. & S.A. AND A. & $\mathbf{N}$ & $N$ & S.A. & S.A. AND A. & SV.A. & S.A. & M.A. & & & $\mathbf{N}$ & M.A. & S.A. AND A. & & $\mathrm{N}$ & \\
\hline & 10 & S.A. AND A. & SV.A. & M.A. & S.A. & S.A. AND A. & SV.A. & S.A. & S.A. & S.A. AND A & SV.A. & M.A. & S.A. & S.A. & SV.A. & & S.A. & S.A. AND A. & SV.A. & S.A. & S.A. \\
\hline & 11 & S.A. AND A. & $\mathrm{N}$ & S.D. & M.D. & V.D. AND D. & S.A. & $\mathbf{N}$ & S.D. & & $\mathrm{N}$ & $\mathrm{N}$ & M.D. & & $\mathbf{N}$ & M.D. & M.D. & EX.D. & $N$ & M.D. & M.D. \\
\hline
\end{tabular}


Table 11. . Niğde, Kulu, Seydisehir,Cumra and Karapınar stations index results -1

\begin{tabular}{|c|c|c|c|c|c|c|c|c|c|c|c|c|c|c|c|c|c|c|c|c|c|}
\hline \multirow{2}{*}{ YEARS } & \multirow{2}{*}{ MONTHS } & \multicolumn{4}{|c|}{ 17250-NIGDE } & \multicolumn{4}{|c|}{ 17754-KULU } & \multicolumn{4}{|c|}{ 17898-SEYDISEHIR } & \multicolumn{4}{|c|}{ 17900-CUMRA } & \multicolumn{4}{|c|}{ 17902-KARAPINAR } \\
\hline & & Al & PNI & SPI & SPEI & AI & PNI & SPI & SPEI & Al & PNI & SPI & SPEI & & PNI & $=$ & & Al & PNI & SPI & SPEI \\
\hline \multirow{11}{*}{1981} & 1 & E. I & & & & E. D. & N. & EO. D. & E. D. & 50 & $\mathbf{N}$. & EO. D. & & $E$ & N & EO. D. & & & $N$ & & \\
\hline & 2 & & 10 & & N & E. & $\mathrm{N}$ & SV. D. & & E. & $\mathbf{N}$. & N. & & & & & & & & & \\
\hline & 3 & & 10 & N. & & VD. & & $\mathrm{N}$. & S. D. & & V.A. A & M. A. & & & N. & $\mathrm{N}$ & E. D. & i.) & 1. A. & A. & \\
\hline & 4 & A. \&. A. & sv. A. & & & S. D. & M. A. & S. A. & S. D. & & SV. A & M. A. & & & SV. A. & & E. D. & & $\mathrm{VA}$ & A. & \\
\hline & 5 & VD. \& D. & $\mathbf{N}$. & EO. D. & M. D. & S. D. & N. & N. & S. A. & & N. & N. & & & N. & N. & E. D. & S.A & S. A. & $\mathbf{N}$. & \\
\hline & 6 & S.A. 8 & & & & S. A & N. & N. & & & & M. D. & & & & & E. D. & S.A. 8 & $\mathbf{N}$. & & \\
\hline & 7 & S.A. 8 & M. A. & & s & S. A & $N$ & EO. D. & S. A. & a. & M. A. & 2 & & & & SV. D. & E. D. & S.A. & V.A. A. & & SV.A \\
\hline & 8 & 5 & $N$ & & M. A. & S. A & SV. A. & S. A. & M. A. & A. & N. & & & S. A. & & $\mathrm{N}$. & E. D. & S. A. & N. & N. & M. A \\
\hline & 8 & S. A. \& & S. A. & $\mathrm{N}$ & S. A. & S. A. \& & SV. A. & S. A. & S. A. & S. A. \& A. & SV. A & M. A. & M. A. & S. A. & M. A. & 2 & E. D. & S. A. \& A. & sv. A. & S. A. & S.A. \\
\hline & & S.A. \& A. & sv. A. & S.A. & S. A. & S. A. \& A. & SV. A. & S. A. & S. A. & S. A. \& A. & SV. A & M. A. & S. A. & S. A. \& A. & SV. A. & M. A. & E. D. & S. A. \& A. & sv. A. & M. A. & \\
\hline & 11 & E. & $\mathbf{N}$. & $N$ & S. D. & E. D. & $\mathrm{N}$. & $\mathrm{N}$. & S. D. & 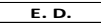 & $N$ & 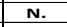 & M. D. & VD. \& D. & M. A. & S. A. & E. D. & VD & A. A. & & \\
\hline
\end{tabular}


Table 12. Niğde, Kulu, Seydisehir,Cumra and Karapınar stations index results -2

\begin{tabular}{|c|c|c|c|c|c|c|c|c|c|}
\hline & & 1725 & $50-\mathrm{NI}$ & IGDE & & 177 & 54-K & KULU & \\
\hline & & & PNI & & SPEI & & PNI & & SPEI \\
\hline & & E. D. & N. & $\mathrm{N}$. & M. D. & E. D. & S. A. & N. & S. D. \\
\hline & 3 & $\begin{array}{l}\text { E.D. } \\
\text { L. D. }\end{array}$ & \begin{tabular}{|l|} 
N. N. \\
M. A.
\end{tabular} & \begin{tabular}{|l|} 
N. \\
M. A.
\end{tabular} & s. D. & E. D. & M. A. & $\begin{array}{l}\text { M.D. } \\
\text { S. A. }\end{array}$ & s. D. \\
\hline & $\begin{array}{l}4 \\
5\end{array}$ & $\begin{array}{l}\text { VD.\&. D. } \\
\text { S.D. (D.) }\end{array}$ & $\begin{array}{l}\text { N. } \\
N .\end{array}$ & \begin{tabular}{|c|} 
M. D. \\
N.
\end{tabular} & \begin{tabular}{|l|} 
S. D. \\
S. D. \\
\end{tabular} & $\begin{array}{l}\text { E. D. } \\
\text { L. D. }\end{array}$ & \begin{tabular}{|l|}
$\mathrm{N}$ \\
$\mathrm{N}$
\end{tabular} & \begin{tabular}{|l|} 
EO. D. \\
M. D.
\end{tabular} & $\begin{array}{l}\text { VD. } \\
\text { S. D. }\end{array}$ \\
\hline & 6 & S. A. \& A. A. & N. & N. & \begin{tabular}{|l|} 
S. A. \\
S. A.
\end{tabular} & & SV. A. & S. A. & M. A. \\
\hline & $\begin{array}{l}7 \\
8\end{array}$ & $\begin{array}{l}\text { S.A. \& A. } \\
\text { S.A. \& A }\end{array}$ & $\begin{array}{l}\text { SV.A. } \\
\text { sv. A. }\end{array}$ & $\frac{N .}{N .}$ & SV.A. & S.A. \&A. & $\begin{array}{l}\text { SV. A. } \\
\text { SV A. }\end{array}$ & M. A. & $\begin{array}{l}\text { SV.A. } \\
\text { SV.A. }\end{array}$ \\
\hline & $\frac{8}{9}$ & $\begin{array}{l}\text { S.A. \& A. } \\
\text { S. A. A. }\end{array}$ & $\begin{array}{l}\text { SV.A. A. } \\
\text { SV. }\end{array}$ & \begin{tabular}{|l} 
N. \\
M. A.
\end{tabular} & \begin{tabular}{|l|} 
M.A. A. \\
S. A.
\end{tabular} & $\begin{array}{l}\text { S. A. \& A. } \\
\text { S. A. A. }\end{array}$ & $\begin{array}{l}\text { SV.A. A. } \\
\text { SV. A. }\end{array}$ & N. A. & $\mid \begin{array}{l}\text { SV. A. } \\
\text { S. A. }\end{array}$ \\
\hline & 10 & L. D. & N. & M. D. & S. D. & S. A. \&A. & N. & N. & S. A. \\
\hline & 11 & VD.\&D. & $\mathrm{N}$. & N. & \begin{tabular}{|l|} 
S. D. \\
\end{tabular} & VD. \& D. & M. A. & S.A. & S.D. \\
\hline & $\frac{12}{1}$ & $\begin{array}{l}\text { E.D. } \\
\text { E.D. }\end{array}$ & $\begin{array}{l}\frac{\text { N. }}{\text { SV.A. }} \text {. } \\
\text { S }\end{array}$ & $\begin{array}{l}\text { N. } \\
\text { M. A. }\end{array}$ & \begin{tabular}{|l|} 
M. D. \\
s. D.
\end{tabular} & $\begin{array}{l}\text { E. D. } \\
\text { E.D. }\end{array}$ & $\begin{array}{l}\text { N. } \\
\text { sV. A. }\end{array}$ & M. A. & $\mid$\begin{tabular}{|l|} 
VD. \\
S.D.
\end{tabular} \\
\hline & $2 \frac{2}{3}$ & $\begin{array}{l}\text { E. D. } \\
\text { E. D }\end{array}$ & N. & \begin{tabular}{|l|} 
S. D. \\
\end{tabular} & \begin{tabular}{|l|} 
M.D. \\
S.D.
\end{tabular} & $\begin{array}{l}\text { E. D. } \\
\text { E. D }\end{array}$ & $\frac{\text { sv. A. }}{\text { N. }}$ & M.A. & S. D. \\
\hline & $\frac{3}{4}$ & S.A. \& A. & $\frac{\text { N. }}{\text { SV. A. }}$ & VA. & \begin{tabular}{|l|} 
S.D. A. \\
S.
\end{tabular} & & sv. A. & VA. & S. A. \\
\hline & 5 & $\begin{array}{l}\text { S. D. (D.) } \\
\text { S. D. }\end{array}$ & $\mathrm{N}$ & \begin{tabular}{|l|} 
S.D. \\
S.
\end{tabular} & S.D. & S. A. \& A & N. & N. & S.A. \\
\hline 1992 & $\frac{6}{7}$ & $\begin{array}{l}\text { S. D. (A.) } \\
\text { S. A. \& A. }\end{array}$ & $\frac{N .}{N .}$ & $\frac{\text { SV.D. }}{\mathrm{N} .}$ & $\begin{array}{l}\text { S.A. A. } \\
\text { M.A. }\end{array}$ & $\begin{array}{l}\text { S. D. (D.) } \\
\text { S.A.\&A }\end{array}$ & $\mathrm{N}$. & VD. & S.A. \\
\hline & 8 & $\begin{array}{l}\text { S.A. A. \& A. } \\
\text { S. A }\end{array}$ & Sv. A. & $\begin{array}{l}\text { N. } \\
\text { N. }\end{array}$ & \begin{tabular}{|c|} 
M.A. \\
M.A.
\end{tabular} & $\begin{array}{l}\text { S.A. \& A. } \\
\text { S. A. \&A. }\end{array}$ & $\begin{array}{l}\text { N. A. } \\
\text { M. A. }\end{array}$ & $\frac{\text { M.D. }}{\text { N. }}$ & M. A. \\
\hline & 9 & S.A. \&.A. & $\begin{array}{l}\text { M.A. } \\
\text { S. }\end{array}$ & N. & \begin{tabular}{|l|} 
S.A. \\
S.A.
\end{tabular} & S. A.\&A. & SV. A. & M. A. & S.A. \\
\hline & $\frac{10}{11}$ & $\begin{array}{l}\text { S.A. \&. A } \\
\text { E. D. }\end{array}$ & S S. A. & $\begin{array}{l}\text { M. A. } \\
\text { S. D. } \\
\end{array}$ & \begin{tabular}{|l|} 
M. A. \\
M. D.
\end{tabular} & $\begin{array}{l}\text { S.A. \&. } \\
\text { E. D. }\end{array}$ & $\frac{\text { SV. A. }}{\text { N. }}$ & $\frac{N}{N}$ & \begin{tabular}{|l|} 
S.A. \\
S. D.
\end{tabular} \\
\hline & 12 & E. D. & N. & S.D. & VD. & E. D. & $N$. & M. D. & VD. \\
\hline & 2 & $\begin{array}{l}\text { E.D. } \\
\text { E.D. }\end{array}$ & $\begin{array}{l}\text { SV.A. A. } \\
\text { S.A. }\end{array}$ & \begin{tabular}{|l|} 
M. A. \\
S. A. \\
\end{tabular} & \begin{tabular}{|l|} 
S. D. \\
s. D.
\end{tabular} & $\begin{array}{l}\text { E. D. } \\
\text { E. D. }\end{array}$ & $\frac{N .}{N .}$ & U. D. & $\begin{array}{l}\text { M. D. } \\
\text { s. D. }\end{array}$ \\
\hline & 3 & & & M. A. & & VD. \&D. & $\mathrm{N}$. & N. & \\
\hline & $\frac{4}{5}$ & $\begin{array}{l}\text { L.D. } \\
\text { S.A.\& }\end{array}$ & \begin{tabular}{|l|} 
N. \\
S.A. \\
\end{tabular} & N. & \begin{tabular}{|l|} 
S.D. \\
S.A.
\end{tabular} & $\begin{array}{l}\text { S.A. \& A. } \\
\text { VD. \&D. }\end{array}$ & $\frac{\text { SV. A. }}{\mathrm{N} .}$ & $\begin{array}{l}\text { M. A. } \\
\text { SV. D. }\end{array}$ & $\begin{array}{l}\text { S.A. } \\
\text { S. D. }\end{array}$ \\
\hline 1993 & 6 & S. A. \& A. & $\mathrm{N}$. & N. & & S. A. \& A. & $\mathrm{N}$ & N. & S. A. \\
\hline & $\begin{array}{l}7 \\
8\end{array}$ & $\begin{array}{l}\text { S.A. \& A. } \\
\text { S.A. \&.A. }\end{array}$ & $\begin{array}{l}\text { SV.A. } \\
\text { sv. A. }\end{array}$ & $\begin{array}{l}\text { S. A. } \\
\text { S.A. }\end{array}$ & \begin{tabular}{|c|} 
M.A. \\
M.A.
\end{tabular} & $\begin{array}{l}\text { S.A.\&A. } \\
\text { S.A. \&A. }\end{array}$ & $\begin{array}{l}\text { N. } \\
\text { sV.A. }\end{array}$ & $\begin{array}{l}\text { VD. } \\
\text { S.A. }\end{array}$ & A. \\
\hline & 9 & S. A. \& A. A. & S. A. & N. & \begin{tabular}{|l|} 
S.A. A. \\
S.
\end{tabular} & $\begin{array}{l}\text { S.A. \& } A \text {. } \\
\text { S. A. A. }\end{array}$ & $\begin{array}{l}\text { SV. A. } \\
\text { SV. A. }\end{array}$ & M. A. & M. A. \\
\hline & $\frac{10}{11}$ & $\begin{array}{l}\text { S.A.\&.A. } \\
\text { E.D. }\end{array}$ & $\frac{\text { SV.A. }}{\mathrm{N} .}$ & M. A. & \begin{tabular}{|l|} 
S.A. A. \\
S.D. \\
\end{tabular} & $\begin{array}{l}\text { S. A. \& A. } \\
\text { E. D. }\end{array}$ & $\frac{\text { SV. A. }}{N \text {. }}$ & M.A. & $\begin{array}{l}\text { S.A. } \\
\text { S.D. }\end{array}$ \\
\hline & $\frac{11}{12}$ & $\mathrm{E}$ & \begin{tabular}{|c|} 
N. \\
M. A.
\end{tabular} & \begin{tabular}{|l|} 
S. A. \\
\end{tabular} & $\begin{array}{l}\text { S.D. } \\
\text { S. D. }\end{array}$ & $\begin{array}{l}\text { E.D. } \\
\text { E. D. }\end{array}$ & \begin{tabular}{|l|}
$\mathrm{N}$ \\
$\mathrm{N}$
\end{tabular} & N. & D. D. \\
\hline & 1 & E. & $\mathrm{N}$. & N. & S. D. & E. D. & $\mathrm{N}$. & M. D. & \\
\hline & 2 & $\begin{array}{l}\text { E.D. } \\
\text { VD. \& }\end{array}$ & $N$. & N. & S. D. & $\begin{array}{l}\text { E. D. } \\
\text { E. D. }\end{array}$ & $\mathrm{N}$. & VD. & M. D. \\
\hline & 4 & $\begin{array}{l}\text { V. D. B. D. } \\
\text { S.D. }\end{array}$ & $\frac{N}{N}$. & & \begin{tabular}{|l|}
$\mid$ S.D. \\
S. D.
\end{tabular} & E.D. & \begin{tabular}{|l|} 
N. \\
$N$.
\end{tabular} & N. & D. \\
\hline & 5 & S. A. \& A. & SV.A. & M. A. & S. A. & S. A. \& A. & M. A. & M. A. & S. A. \\
\hline & $\frac{6}{7}$ & $\begin{array}{l}\text { S. A. \& A. } \\
\text { S. A \& A. }\end{array}$ & $\frac{\text { S. A. }}{\text { S. A. }}$ & \begin{tabular}{|l|} 
M. A. \\
N.
\end{tabular} & \begin{tabular}{|l|} 
M. A. \\
sV.A.
\end{tabular} & $\begin{array}{l}\text { S. A. \& A. } \\
\text { S.A. \& A. }\end{array}$ & \begin{tabular}{|l|} 
\\
$N$
\end{tabular} & $\begin{array}{l}\mathrm{N} . \\
\mathrm{VD}\end{array}$ & A. \\
\hline & 8 & $\begin{array}{l}\text { S. A. \& A. } \\
\text { S. }\end{array}$ & $\begin{array}{l}\text { S.A. } \\
\text { SV. A. }\end{array}$ & $\begin{array}{l}\text { S. A. } \\
\end{array}$ & M. A. & S. A. \& A. & SV. A. & N. & M. A. \\
\hline & $\frac{9}{10}$ & $\begin{array}{l}\text { S. A. \& A. } \\
\text { S. A. \& A. }\end{array}$ & $\frac{N .}{N .}$ & $\frac{N .}{N}$ & \begin{tabular}{|l|} 
M. A. \\
s. A.
\end{tabular} & $\begin{array}{l}\text { S. A. \&. } \\
\text { L. D. }\end{array}$ & V. A. & $\begin{array}{l}\text { M. A. } \\
\text { M.D. }\end{array}$ & D. 1 \\
\hline & 11 & & S. A. & N. & \begin{tabular}{|l|} 
S. D. \\
\end{tabular} & & N. & N. & \\
\hline & 12 & E. & N. & $\mathrm{N}$. & & E. D. & S.A. & & \\
\hline & 1 & & & N. & S.D. & & & N. & \\
\hline & 2 & S. A. \&A & SV.A. A. & VA. & \begin{tabular}{|l|} 
S. D. \\
\end{tabular} & E. D & SV. A. & M. A. & D. 1 \\
\hline & $\frac{3}{4}$ & E. & $N$. & M. D. & M.D. & E. D. & $\mathrm{N}$. & S. D. & S. D. \\
\hline & $\frac{4}{5}$ & $\begin{array}{l}\text { VD. \& D. } \\
\text { S. D. (A.) }\end{array}$ & $\frac{N .}{N .}$ & $\begin{array}{l}\text { VD. } \\
\text { N. }\end{array}$ & \begin{tabular}{|l|} 
M.D. \\
S. A.
\end{tabular} & $\begin{array}{l}\text { VD. \&D. } \\
\text { S. A. \& A. }\end{array}$ & $\begin{array}{l}\text { N. } \\
\text { sV. A. }\end{array}$ & $\begin{array}{l}\text { S.D. } \\
\text { M. A. }\end{array}$ & D. \\
\hline 1995 & 6 & S.A.\&. A. & N. & M. D. & S. A. & S. A. \& A. & N. & N. & A. \\
\hline & $\frac{7}{8}$ & $\begin{array}{l}\text { S. A. \& A. } \\
\text { S. A. \& A. }\end{array}$ & sv. A. & $\frac{N .}{\text { SV. }}$ & \begin{tabular}{|l|} 
M. A. A. \\
M. A.
\end{tabular} & $\begin{array}{l}\text { S.A. \& } \\
\text { S.A. \& }\end{array}$ & $\begin{array}{l}\text { N. } \\
\text { M. A. }\end{array}$ & $\frac{\text { SV. D }}{N .}$ & A. A. \\
\hline & 9 & S.A.\&.A. & M. A. & $\mathrm{N}$. & S. A. & S.A. 8 & $N$ & $\mathrm{~N}$. & S. A. \\
\hline & 10 & S. D. (D.) & N. & N. & S.D. & S. D. & $\mathrm{N}$. & $\mathrm{N}$. & S.D. \\
\hline & $\frac{11}{12}$ & E. & N. A. & $\begin{array}{l}\text { S.D. } \\
\text { S.A. }\end{array}$ & M. D. & E. & N. & VD. & \\
\hline & 1 & E. & N. & N. & S. D. & $\mathrm{E}$. & $\mathrm{N}$. & $\mathrm{N}$. & S. D. \\
\hline & 3 & & \begin{tabular}{|c|} 
M. A. \\
N.
\end{tabular} & $\begin{array}{l}\text { S. A. } \\
\text { V D. }\end{array}$ & \begin{tabular}{|l|} 
S.D. D. \\
M.D.
\end{tabular} & $\begin{array}{l}\text { E.D. } \\
\text { E. D. }\end{array}$ & N. & $\frac{\mathrm{N} .}{\mathrm{EO} . \mathrm{D} .}$ & \\
\hline & 4 & VD.\&D. & N. & M. D. & S. D. & VD. \& & $\mathrm{N}$. & N. & S. D. \\
\hline & 6 & S.A. & $\begin{array}{l}\text { S. A. } \\
\text { sv.A. }\end{array}$ & S.A. & $\mid$\begin{tabular}{|c|} 
S. A. \\
M.A.
\end{tabular} & $\begin{array}{l}\text { S.A. } 8 \\
\text { S.A. } 8\end{array}$ & SV. A. & S. A. & At \\
\hline 19 & 7 & S. A. & SV. A. & S. A. & sv. A. & S. A. \& & sV. A. & S. A. & s \\
\hline & 8 & $\begin{array}{l}\text { S. A. \& A. } \\
\text { S. A. \& A. }\end{array}$ & SV. A. & S. A. & sv. A. & $\begin{array}{l}\text { S.A.\& } \\
\text { S.A. \& }\end{array}$ & N. & N. & \\
\hline & 10 & $\mathrm{~L}$. & N. & N. & \begin{tabular}{|l|} 
S.A. D. \\
S.
\end{tabular} & L.D. & N. & M.D. & \\
\hline & 11 & S.A. \&.A. & Sv. A. & VA. & S. D. & S.A.\&A & SV. A. & VA. & S.A. \\
\hline & 1 & & & $\begin{array}{l}\text { M.D } \\
\text { N. }\end{array}$ & & & A. & $\begin{array}{l}\text { S. D. } \\
\text { S. A. }\end{array}$ & \\
\hline & 2 & E. & $N$ & N. & S.D & $\mathrm{E}$ & $\mathrm{N}$ & N. & \\
\hline & $\frac{3}{4}$ & E.I & \begin{tabular}{|l|} 
M. A. \\
S.A.
\end{tabular} & S.A. & s. & $\begin{array}{l}\text { E. D. } \\
\text { E. D. }\end{array}$ & N. & $\frac{\mathrm{N} .}{\mathrm{VD}}$ & \\
\hline & 5 & S.D & N. & $\mathrm{N}$. & \begin{tabular}{|l|} 
S. A. \\
\end{tabular} & L.D. & $\mathrm{N}$ & SV. D. & S.D. \\
\hline 1997 & $\frac{6}{7}$ & & $\frac{\text { N. }}{\text { SN. A. }}$ & \begin{tabular}{|l|} 
N. \\
S.A.
\end{tabular} & M.A. & S. A. & $\frac{N .}{\text { sV.A. }}$ & \begin{tabular}{|l|} 
S.D. \\
M.A.
\end{tabular} & s. \\
\hline & 8 & & $\frac{N}{N}$ & & SV.A. & S. A. & $\frac{S V . A}{N .}$ & \begin{tabular}{|l|} 
M. A. \\
M. D.
\end{tabular} & M. A. \\
\hline & 9 & S.A. \&.A. & N. & M. D. & S. A. & S. A. \& A. & SV. A. & S. A. & S. A. \\
\hline & $\frac{10}{11}$ & $\begin{array}{l}\text { VD. } \\
\text { S.A. }\end{array}$ & $\begin{array}{l}\frac{\text { N. }}{\text { Sv. A. }} \\
\text { Sill }\end{array}$ & & & $\begin{aligned} V D \\
E\end{aligned}$ & $\begin{array}{l}\mathrm{N} . \\
\mathrm{N} .\end{array}$ & E.D. & \\
\hline & 12 & & $N$ & vi & $\mathrm{v}$ & & $\mathrm{N}$ & S. D. & \\
\hline & 2 & & $\frac{N}{N .}$ & $N$ & & $\begin{array}{ll}\text { E. } \\
\text { E. }\end{array}$ & $\begin{array}{l}\text { SV. A. } \\
\text { S.A. }\end{array}$ & M. A. & $\begin{array}{l}\text { S. D. } \\
\text { S.D. }\end{array}$ \\
\hline & 3 & 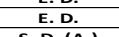 & $N$. & M. D. & M. D. & E.I & N. & VD. & s. \\
\hline & $\begin{array}{l}4 \\
5\end{array}$ & $\frac{5}{5}$ & S.A & s. & \begin{tabular}{|l|}
$\mid$ S.A. A. \\
S.A.
\end{tabular} & $\begin{array}{l}\text { VD. } \\
\text { VD. }\end{array}$ & N. & $\begin{array}{l}\text { U. U. D. } \\
\text { V. D. }\end{array}$ & \\
\hline $19 \mathrm{~s}$ & 6 & & \begin{tabular}{|l}
$\mathbf{N}$ \\
\end{tabular} & M.D. & S.A. & S. A. & S.A. & N. & \\
\hline & & & Sv. A. & S. A. & SV.A. & & $\mathrm{N}$. & N. & \\
\hline & & & & & & & & & \\
\hline & 9 & & SV.A. A. & & & & 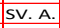 & N. & \\
\hline & 10 & $L$ & N. & M. D. & S. D. & S. A. & A. & M. A. & \\
\hline & 11 & & $\begin{array}{l}\text { SV. A. } \\
\text { N. }\end{array}$ & & $\begin{array}{l}\text { S.A. } \\
\text { VD. }\end{array}$ & & & N. & \\
\hline & 1 & & SV.A. A. & M. & s. & & SV. A. & S. A. & \\
\hline & & & $N$. & & s. & & 15 & $N$. & \\
\hline & 4 & & N. & & & S. D. & & & \\
\hline & 5 & S. A. & SV. A. & M. A. & S. A. & S.A. & sv. A. & M. A. & 5. \\
\hline & 7 & & \begin{tabular}{|c|} 
N. \\
sv. A.
\end{tabular} & E. & & & N. & & \\
\hline & 8 & S. A. \& A. & SV. A. & S. A. & SV.A. & S. A. & $\mathrm{N}$. & O. D. & S. A. \\
\hline & $\frac{9}{10}$ & & SV.A. & $s$. & & & \begin{tabular}{|l|} 
S. A. \\
$N$
\end{tabular} & N. & \\
\hline & $\frac{10}{11}$ & & A. & $\frac{1}{v}$ & 5. & & & M. A. & \\
\hline & & & & & & & & & \\
\hline & 1 & & N. & EOOD. & VD. & & $\mathrm{N}$ & EO D. D. & \\
\hline & & & $\mathrm{N}$ & M. D. & & & . & S.D. & \\
\hline & 3 & E. D. & $N$ & N. & S. D. & E.I & M. A. & S. A. & D. \\
\hline & $\frac{4}{5}$ & & $\frac{N}{N}$ & $N$ & S. A. & & $\mathrm{N}$ & N. & \\
\hline 2000 & 6 & S.A.\&.A. & $\mathrm{N}$. & M. D. & S. A. & S. A & N. & $\mathrm{N}$. & S.A. \\
\hline & 8 & $\begin{array}{l}\text { S.A. } \\
\text { S. A. \& }\end{array}$ & $\begin{array}{l}\text { SS. A. } \\
\text { sv. A. }\end{array}$ & S. A. & $\mid \begin{array}{l}\text { SV. A. } \\
\text { M. A. }\end{array}$ & $\begin{array}{l}\text { S. A. } \\
\text { S. A. }\end{array}$ & $\frac{\mathrm{N}}{\mathrm{N}}$ & $\begin{array}{l}\text { N. } \\
\text { S. D. }\end{array}$ & \begin{tabular}{|l|} 
S. A. A. \\
M. A.
\end{tabular} \\
\hline & 9 & S. A. \& & SV. A. & S. A. & \begin{tabular}{|l|} 
S. A. \\
\end{tabular} & S. A. & A. & S. A. & M. \\
\hline & $\frac{10}{11}$ & S.A. & N. & VA & $\mid$\begin{tabular}{|l|} 
S. D. \\
S. D.
\end{tabular} & $\begin{array}{l}\text { S.A. \& } \\
\text { S.D. }\end{array}$ & $\begin{array}{l}\text { S. A. } \\
\text { SV.A. }\end{array}$ & N. & \\
\hline & $\frac{11}{12}$ & & 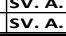 & & \begin{tabular}{|l|} 
S.D. \\
S.D. \\
\end{tabular} & S. D. D & $\begin{array}{l}\text { SV. A. } \\
\text { S. A. }\end{array}$ & $\begin{array}{l}\text { M. A. } \\
\text { S. A. }\end{array}$ & \\
\hline
\end{tabular}


Table 13. Niğde, Kulu, Seydisehir,Cumra and Karapınar stations index results-3

\begin{tabular}{|c|c|c|c|c|c|c|c|c|c|c|}
\hline & & 1725 & OO-NI & GDE & & 177 & 54-K! & KULU & & 17898 \\
\hline & & Al & PNI & & SPEI & $=$ & PNI & SPI & & Al \\
\hline & & S. A.\&.A. & SV.A. & \begin{tabular}{|l|} 
SV.A. \\
SV.
\end{tabular} & $\begin{array}{l}\text { S. D. } \\
\text {. }\end{array}$ & L. D. & V. A. & VA. & S. D. & E.D. \\
\hline & 3 & S. D. (D.) & \begin{tabular}{|l|} 
N. \\
N.
\end{tabular} & $\begin{array}{l}\text { Sv. D. } \\
\text { N. }\end{array}$ & $\begin{array}{l}\text { s. D. } \\
\end{array}$ & $\begin{array}{l}\text { E. D. } \\
\text { S. D. (A.) }\end{array}$ & M. A. & S. A. & S. A. & $\begin{array}{l}\text { E.D. } \\
\text { S.A. \&. A. }\end{array}$ \\
\hline & $\begin{array}{l}4 \\
5\end{array}$ & $\begin{array}{l}\text { S. D. (A.) } \\
\text { VDD. \&. D. }\end{array}$ & \begin{tabular}{|l|} 
S.A. \\
N.
\end{tabular} & \begin{tabular}{|l|} 
S.A. \\
M.D.
\end{tabular} & $\begin{array}{l}\text { S.A. A. } \\
\text { S.D. }\end{array}$ & $\begin{array}{l}\text { S.A. \& A. } \\
\text { VD. \& D. }\end{array}$ & $\frac{\text { sV. A. }}{\mathrm{N} \text {. }}$ & $\begin{array}{l}\text { M.A. } \\
\text { VD. }\end{array}$ & $\mid \begin{array}{l}\text { S. A. } \\
\text { S.D. }\end{array}$ & $\begin{array}{l}\text { VD.\&D. } \\
\text { VD.\&D. }\end{array}$ \\
\hline 2001 & 6 & S. A. \& A. & Sv. A. & M. A. & M. A. & S. A. \& A. & SV.A. & M. A. & M. A. & $\begin{array}{l}\text { SD. \& D. } \\
\text { S.A. A. }\end{array}$ \\
\hline & $\begin{array}{l}7 \\
8\end{array}$ & S. A. \& A. & SV.A. & \begin{tabular}{|l|} 
S.A. \\
S.A \\
\end{tabular} & sv.A. & $\begin{array}{l}\text { S.A. \&A. } \\
\text { S.A. \& A. }\end{array}$ & $\begin{array}{l}\text { SV.A. } \\
\text { SV.A. }\end{array}$ & $\begin{array}{l}\text { S.A. } \\
\text { S.A. }\end{array}$ & \begin{tabular}{|l|} 
SV.A. \\
SV.
\end{tabular} & $\begin{array}{l}\text { S.A. \&.A. } \\
\text { S.A. \& A. }\end{array}$ \\
\hline & 9 & S.A. \& A. & SV.A. & \begin{tabular}{|l|} 
S.A. A. \\
\end{tabular} & M. A. & $\begin{array}{l}\text { S. A. \&A. } \\
\text { S. A. \&A. }\end{array}$ & S. A. & N. & S. A. & S.A.\&A. A. \\
\hline & 10 & S. A. \& A. & S. A. & N. & S. A. & S. A. \& A. & SV. A. & M. A. & S. A. & S.A.\&A. \\
\hline & 11 & E. D. & $\mathbf{N}$. & M. D. & M. D. & E. D. & N. & EO. D. & VD. & E.D. \\
\hline & $\frac{12}{1}$ & $\begin{array}{l}\text { E.D. } \\
\text { E.D. }\end{array}$ & N. & \begin{tabular}{|l|}
$\mathbf{N}$. \\
$\mathbf{N}$
\end{tabular} & \begin{tabular}{|l|} 
S.D. D. \\
M. D.
\end{tabular} & $\begin{array}{l}\text { E. D. } \\
\text { E. D. }\end{array}$ & $\frac{N .}{N}$. & EO. D. & $\begin{array}{l}\text { E. D. } \\
\text { M. D. }\end{array}$ & $\begin{array}{l}\text { E.D. } \\
\text { E.D. }\end{array}$ \\
\hline & 2 & VD.\& D. & SV. A. & M. A. & S. D. & E. D. & SV.A. & M. A. & S. D. & E. D. \\
\hline & $\begin{array}{l}3 \\
4\end{array}$ & S.D. (A.) & $\frac{\text { SV. A. }}{\mathrm{N} .}$ & \begin{tabular}{|l|} 
M. A. \\
EO. D.
\end{tabular} & \begin{tabular}{|l|} 
S. D. \\
M.. D.
\end{tabular} & $\begin{array}{l}\text { L. D. } \\
\text { E.D. }\end{array}$ & $\begin{array}{l}\text { S.A. } \\
\text { N. }\end{array}$ & $\begin{array}{l}\text { S. A. } \\
\text { M. D. }\end{array}$ & \begin{tabular}{|l|} 
S. D. \\
M. D.
\end{tabular} & $\begin{array}{l}\text { E.D. } \\
\text { E.D. }\end{array}$ \\
\hline & 5 & S. D. (D.) & N. & N. & \begin{tabular}{|l|} 
S. D. \\
\end{tabular} & S. A. \& A. & S.A. & S. A. & S. A. & S. A. \& A. \\
\hline 2002 & $\frac{6}{7}$ & $\begin{array}{l}\text { S.A. \& A. } \\
\text { S.A. \&.A. }\end{array}$ & M.A. & \begin{tabular}{|l|} 
S.A. \\
SV.D.
\end{tabular} & M.A. & $\begin{array}{l}\text { S.A. \& A. } \\
\text { S.A. \&A }\end{array}$ & $\frac{\text { SV.A. }}{\mathrm{N} .}$ & $\begin{array}{ll}\text { M.A. } \\
\text { N. }\end{array}$ & $\begin{array}{l}\text { M.A. } \\
\text { SV.A. }\end{array}$ & $\begin{array}{l}\text { S. A. \& A. } \\
\text { S.A. \& A. }\end{array}$ \\
\hline & $\frac{7}{8}$ & $\begin{array}{l}\text { S. A. \& A. } \\
\text { S. A. \& A. }\end{array}$ & \begin{tabular}{|l|}
$\mathbf{N}$. \\
$\mathbf{N}$. \\
\end{tabular} & $\begin{array}{l}\text { SV.D. } \\
\text { SV.D. }\end{array}$ & $\begin{array}{l}\text { M.A. } \\
\text { M. A. }\end{array}$ & $\begin{array}{l}\text { S. A. \& A. } \\
\text { S. A. A A. }\end{array}$ & N. & $\mid \begin{array}{l}\mathrm{N} . \\
\mathrm{N} .\end{array}$ & $\begin{array}{l}\text { SV. A. } \\
\text { M. A. }\end{array}$ & $\begin{array}{l}\text { S. A. \& A. } \\
\text { S. A. \& A. }\end{array}$ \\
\hline & $\frac{9}{10}$ & $\begin{array}{l}\text { S.A. \& A. } \\
\text { S.A.\&AA }\end{array}$ & N. & EO. D. & \begin{tabular}{|l|} 
S.A. A. \\
S.A.
\end{tabular} & $\begin{array}{l}\text { S.A. \& A. } \\
\text { S.A. \& A }\end{array}$ & $\begin{array}{ll}N . \\
N\end{array}$ & M. D. & S.A. & S. D. (A.) \\
\hline & $\frac{10}{11}$ & $\begin{array}{l}\text { S. A. \& A. } \\
\text { V D. \&. D. }\end{array}$ & $\begin{array}{l}\text { S. A. } \\
\text { N. }\end{array}$ & $\begin{array}{l}\mathrm{N} . \\
\mathrm{N} .\end{array}$ & $\begin{array}{l}\text { S. A. } \\
\text { S. D. }\end{array}$ & $\begin{array}{l}\text { S. A. \& A. } \\
\text { VD. \& D. }\end{array}$ & $\begin{array}{l}\text { SV. A. } \\
\text { S. A. }\end{array}$ & $\begin{array}{l}\text { M. A. } \\
\text { S. A. }\end{array}$ & $\begin{array}{l}\text { S. A. } \\
\text { S. D. }\end{array}$ & $\begin{array}{c}\text { S.D. (A.) } \\
\text { E. D. }\end{array}$ \\
\hline & 12 & E. D. & N. & N. & M. D. & E. D. & SV.A. & M. A. & S. D. & E. D. \\
\hline & $\frac{1}{2}$ & $\begin{array}{l}\text { E.D. } \\
\text { E.D. }\end{array}$ & $\frac{N .}{N .}$ & $\frac{\mathrm{N} .}{\mathrm{VD} .}$ & $\begin{array}{l}\text { S.D. } \\
\text { M. D. }\end{array}$ & $\begin{array}{l}\text { E. D. } \\
\text { E.D. }\end{array}$ & $\frac{N .}{N .}$ & $\begin{array}{l}\mathrm{N} . \\
\mathrm{V} . \\
\end{array}$ & $\begin{array}{l}\text { S. D. } \\
\text { M.D. }\end{array}$ & $\begin{array}{l}\text { E.D. } \\
\text { E.D. }\end{array}$ \\
\hline & 3 & E.D. & N. & N. & M.D. & E. D. & $\frac{N}{N}$ & $\mathrm{~N}$. & $\begin{array}{l}\text { S.D. D. } \\
\text { S. D. }\end{array}$ & $\begin{array}{l}\text { E.D. } \\
\text { E.D. }\end{array}$ \\
\hline & $\begin{array}{l}4 \\
5\end{array}$ & VD.\&D. & N. & $N$. & S.D. & VD. \& D. & $\mathrm{N}$. & $N$. & S. D. & VD.\&D. \\
\hline 2003 & $\frac{5}{6}$ & $\begin{array}{l}\text { S. A. \& A. } \\
\text { S. A. \& A. }\end{array}$ & $\begin{array}{l}\text { N. } \\
\text { Sv. A. }\end{array}$ & $\begin{array}{l}\text { N. A. } \\
\text { s. }\end{array}$ & $\begin{array}{l}\text { S.A. A. } \\
\text { M.A. }\end{array}$ & $\begin{array}{l}\text { S.D. (A.) } \\
\text { S. A. \& A. }\end{array}$ & $\frac{\text { N. }}{\text { SV. A. }}$ & M. A. & $\begin{array}{l}\text { S.A. A. } \\
\text { M. A. }\end{array}$ & $\begin{array}{l}\text { S.A. \& A. } \\
\text { S. A. \& A. }\end{array}$ \\
\hline & 7 & S. A. \& A. & SV.A. & S. A. & sv.A. & S. A. \& A. & N. & N. & M.A. & S.A. \&.A. \\
\hline & $\frac{8}{9}$ & $\begin{array}{l}\text { S. A. \& A. } \\
\text { S. A. A. A. }\end{array}$ & $\frac{\text { Sv. A. }}{\text { N. }}$ & $\begin{array}{l}\text { S. A. } \\
\text { N. }\end{array}$ & $\begin{array}{l}\text { SV. A. } \\
\text { S. A. }\end{array}$ & $\begin{array}{l}\text { S. A. \& A. } \\
\text { L. D. }\end{array}$ & $\begin{array}{l}\text { SV. A. } \\
\text { N. }\end{array}$ & $\begin{array}{l}\text { S. A. } \\
\text { EO. D. }\end{array}$ & $\begin{array}{l}\text { SV. A. } \\
\text { S. D. }\end{array}$ & $\begin{array}{l}\text { S. A. \& A. } \\
\text { S. D. (A.) }\end{array}$ \\
\hline & 10 & S. D. (A.) & $\mathrm{N}$. & N. & S. A. & S. A. \& A. & SV.A. & S. A. & S. A. & S. D. (A.) \\
\hline & $\frac{11}{12}$ & $\begin{array}{l}\text { E.D. } \\
\text { E.D. }\end{array}$ & $\begin{array}{l}\text { N. } \\
\text { sV. A. }\end{array}$ & $\begin{array}{l}\text { N. } \\
\text { s. A. }\end{array}$ & $\begin{array}{l}\text { M. D. } \\
\text { S. D. }\end{array}$ & $\begin{array}{l}\text { L.D. } \\
\text { E. D. }\end{array}$ & $\begin{array}{l}\text { SV. A. } \\
\text { N. }\end{array}$ & $\begin{array}{c}\text { M. A. } \\
\text { N. }\end{array}$ & $\begin{array}{l}\text { S. D. } \\
\text { M. D. }\end{array}$ & VD. \& D. \\
\hline & 1 & E. D. & N. & N. & M. D. & E. D. & $\mathrm{N}$. & N. & M. D. & E.D. \\
\hline & 2 & $\begin{array}{l}\text { E.D. } \\
\text { L.D. }\end{array}$ & SV.A. & M. A. & S. D. & VD. \& D. & $\begin{array}{l}\text { SV.A. } \\
\text { SV.A. }\end{array}$ & VA. & S. D. & E.D. \\
\hline & 4 & VD. \&. D. & N. A. & \begin{tabular}{|l|} 
M. A. \\
S. D.
\end{tabular} & \begin{tabular}{|l|} 
S.D. \\
S. D. \\
\end{tabular} & S.D. (D.) & \begin{tabular}{|l|} 
S. A. A. \\
M. A.
\end{tabular} & $\begin{array}{l}\text { M. A. } \\
\text { S. A. }\end{array}$ & S.D. & $\begin{array}{l}\text { D. Q D. } \\
\text { L.D. }\end{array}$ \\
\hline & 5 & S. A. \& A. & SV. A. & M. A. & S. A. & S. A. \& A. & SV.A. & M. A. & S. A. & S. D. (A.) \\
\hline 2004 & $\frac{6}{7}$ & $\begin{array}{l}\text { S. A. \& A. } \\
\text { S. A. \& A. }\end{array}$ & $\frac{\text { Sv. A. }}{\text { N. }}$ & \begin{tabular}{|l|} 
S.A. \\
M.D.
\end{tabular} & \begin{tabular}{|l|} 
M.A. A. \\
M. A.
\end{tabular} & $\begin{array}{l}\text { S. A. \& A. } \\
\text { S. A. \& A. }\end{array}$ & N. & $\begin{array}{l}\text { S. D. } \\
\text { N. }\end{array}$ & $\mid$\begin{tabular}{|l} 
S. A. \\
M. A.
\end{tabular} & $\begin{array}{l}\text { S. A. \& A. } \\
\text { S. A. A. }\end{array}$ \\
\hline & 8 & S.A. \& A. & SV. A. & $\begin{array}{l}\text { S. A. } \\
\text { M. }\end{array}$ & SV.A. & S. A. \& A. & M. A. & $\frac{N}{N}$ & SV.A. & S.A.\&A. \\
\hline & $\frac{9}{10}$ & $\begin{array}{l}\text { S. A. \& A. } \\
\text { S. A. \& A. }\end{array}$ & $\begin{array}{l}\text { N. } \\
\text { sV. A. }\end{array}$ & \begin{tabular}{|l|} 
M. D. \\
S.A.
\end{tabular} & $\begin{array}{l}\text { S. A. } \\
\text { S.A. }\end{array}$ & $\begin{array}{l}\text { S. D. (A.) } \\
\text { S.A. \& A. }\end{array}$ & $\frac{\text { N. }}{\text { SV. A. }}$ & $\frac{\text { SV. D. }}{N .}$ & $\begin{array}{l}\text { S. A. } \\
\text { S. A. }\end{array}$ & $\begin{array}{l}\text { S. A. \& A. } \\
\text { S. A. A. }\end{array}$ \\
\hline & 11 & E. D. & N. & S. D. & & E. D. & N. & $N$ & S. A. & E. D. \\
\hline & 12 & & SV.A. & M. A. & S. D. & & SV.A. & VA. & D. & E. \\
\hline & 1 & E. & N. & N. & M. D. & C.D. & SV.A. & M. A. & D. & E. \\
\hline & 2 & E. & N. & N. & S. D. & E. D. & N. & N. & U. D. & E. \\
\hline & $\begin{array}{l}3 \\
4\end{array}$ & $\begin{array}{l}\text { E. D. } \\
\text { S. D. (A. }\end{array}$ & \begin{tabular}{|l|} 
N. \\
s.A.
\end{tabular} & \begin{tabular}{|l|} 
M. D. \\
S.A.
\end{tabular} & \begin{tabular}{|l|} 
M. D. \\
s. D.
\end{tabular} & $\begin{array}{l}\text { E. D. } \\
\text { VDD \& }\end{array}$ & $\frac{N .}{N}$ & \begin{tabular}{|l|} 
M. D. \\
s. D.
\end{tabular} & $\begin{array}{l}\text { S. D. } \\
\text { S. D. }\end{array}$ & $\begin{array}{l}\text { E.D. } \\
\text { E.D. }\end{array}$ \\
\hline & 5 & S. A. \& A. & SV. A. & M. A. & S. A. & S. D. (A.) & $\mathrm{N}$. & N. & S. A. & S. A. \& A. A. \\
\hline 2005 & 6 & $\begin{array}{l}\text { S.A. \& A. } \\
\text { S.A. B A }\end{array}$ & \begin{tabular}{|l} 
M.A. \\
SS.A.
\end{tabular} & \begin{tabular}{|l|} 
S.A. \\
S.A
\end{tabular} & \begin{tabular}{|c|} 
M.A.A. \\
SY.A
\end{tabular} & $\begin{array}{l}\text { S.A. \& A. } \\
\text { S.A\&A }\end{array}$ & N. & $\mathrm{N}$. & 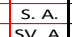 & $\begin{array}{l}\text { S.A. \& A. } \\
\text { S.A. \& A }\end{array}$ \\
\hline & 8 & S. A. \& A. & SV. A. & \begin{tabular}{|l|} 
S. A. \\
\end{tabular} & sv. A. & S. A. \& A. & $\mathrm{N}$. & $\mathrm{N}$ & SV. A. & S.A.\&A. \\
\hline & 9 & S. A. \& A. & N. & EO. D. & S. A. & S. A. \& A. & N. & S. D. & S.A. & S.A. \& A. \\
\hline & $\begin{array}{l}10 \\
11\end{array}$ & $\begin{array}{l}\text { S. D. (A.) } \\
\text { V D. D. }\end{array}$ & $\begin{array}{l}\text { S. A. } \\
\text { SV.A. }\end{array}$ & \begin{tabular}{|l|} 
N. A. \\
S. \\
\end{tabular} & \begin{tabular}{|l|} 
S.D. \\
S.D.
\end{tabular} & $\begin{array}{l}\text { S. D. (A.) } \\
\text { E. D. }\end{array}$ & $\begin{array}{l}\text { S. A. } \\
\text { N. }\end{array}$ & \begin{tabular}{|l|} 
N. \\
S. D.
\end{tabular} & $\begin{array}{l}\text { S.D. } \\
\text { M. D. }\end{array}$ & $\begin{array}{r}\text { VD. \&D. } \\
\text { E.D. }\end{array}$ \\
\hline & 12 & E. D. & SV. A. & \begin{tabular}{|l|} 
S. A. \\
\end{tabular} & S. D. & E. D. & SV.A. & VA. & S. D. & E. D. \\
\hline & 1 & E. & $\begin{array}{l}\text { N. } \\
\text { S. }\end{array}$ & N. & S. D. & E. & N. & N. & M. D. & E. \\
\hline & $\begin{array}{l}2 \\
3\end{array}$ & $\begin{array}{l}\text { E.D. } \\
\text { VD. \&. D. }\end{array}$ & S.A. & $\begin{array}{l}\text { S.A. } \\
\text { N. }\end{array}$ & $\begin{array}{l}\text { S.D. } \\
\text { S. D. }\end{array}$ & $\begin{array}{l}\text { E. D. } \\
\text { VD. \&. }\end{array}$ & $\frac{\text { SV.A. }}{\text { N. }}$ & $\begin{array}{c}\text { M. A. } \\
\text { N. }\end{array}$ & $\begin{array}{l}\text { S.D. D. } \\
\text { S. D. }\end{array}$ & $\begin{array}{l}\text { E.D. } \\
\text { E.D. }\end{array}$ \\
\hline & $\begin{array}{l}4 \\
5 \\
\end{array}$ & $\begin{array}{l}\text { L.D. } \\
\text { S. D. (A.) }\end{array}$ & $\frac{N .}{N .}$ & N. & $\begin{array}{l}\text { S. D. } \\
\text { S.A. }\end{array}$ & $\begin{array}{l}\text { S.A. \& A. } \\
\text { S.D. (A.) }\end{array}$ & $\frac{\text { SV.A. }}{\mathrm{N} .}$ & M.A. & $\begin{array}{l}\text { S.A. A. } \\
\text { S.A. }\end{array}$ & $\begin{array}{l}\text { S. D. (A.) } \\
\text { S. A. \&. A. }\end{array}$ \\
\hline 2006 & 6 & S. A. \& A. & N. & N. & M. A. & S. A. \& A. & N. & S. D. & S. A. & $\begin{array}{l}\text { S.A. A. \& A. } \\
\text { S. A }\end{array}$ \\
\hline & $\begin{array}{l}7 \\
8\end{array}$ & $\begin{array}{l}\text { S. A. \& A. A. } \\
\text { S. A. \& A A. }\end{array}$ & $\frac{\text { SV. A. }}{\mathrm{N} .}$ & $\frac{N .}{N}$ & \begin{tabular}{|l|} 
SV.A. \\
sV.A.
\end{tabular} & $\begin{array}{l}\text { S. A. \& A. } \\
\text { S.A. \& A. }\end{array}$ & $\frac{\text { sV. A. }}{\mathrm{N} \text {. }}$ & M.A. & $\begin{array}{l}\text { SV.A.j } \\
\text { SV.A.j }\end{array}$ & $\begin{array}{l}\text { S.A. \& A. } \\
\text { S.A. \& A. }\end{array}$ \\
\hline & $\frac{8}{9}$ & $\begin{array}{l}\text { S.A. A. A. } \\
\text { S. A. A. }\end{array}$ & $\begin{array}{l}\text { SN. A. } \\
\text { Sv. }\end{array}$ & \begin{tabular}{|l|} 
S. A. \\
\end{tabular} & $\begin{array}{l}\text { SV. A. } \\
\text { S. A. }\end{array}$ & $\begin{array}{l}\text { S.A. A A. } \\
\text { S. D. (D.) }\end{array}$ & N. & EO. D. & $\begin{array}{l}\text { S. A. } \\
\text { S. }\end{array}$ & $\begin{array}{l}\text { S.A. A. A. } \\
\text { S. A. A. }\end{array}$ \\
\hline & 10 & VD. \& D. & N. & SV.D. & S. D. & VD. \& D. & N. & M. D. & S. D. & E. \\
\hline & 11 & $\begin{array}{l}\text { E.D. } \\
\text { E.D. }\end{array}$ & \begin{tabular}{|c|} 
N. \\
Sv. A.
\end{tabular} & \begin{tabular}{|l|} 
N. \\
S.A.
\end{tabular} & $\begin{array}{l}\text { S.D. } \\
\text { S.D. }\end{array}$ & $\begin{array}{l}\text { E. D. } \\
\text { E. D }\end{array}$ & $\begin{array}{l}\text { SV.A. } \\
\text { SV.A. }\end{array}$ & $\begin{array}{l}\text { M.A. } \\
\text { SV.A. }\end{array}$ & $\begin{array}{l}\text { S.D. } \\
\text { S.D. }\end{array}$ & E.D. \\
\hline & 12 & $\begin{array}{l}\text { E.D. } \\
\text { E.D. }\end{array}$ & $\begin{array}{l}\text { MV. A. } \\
\text { M. A. }\end{array}$ & $\begin{array}{l}\text { SV.A. A. } \\
\text { S. A. }\end{array}$ & \begin{tabular}{|l|} 
S.D. \\
S. D. \\
\end{tabular} & $\begin{array}{l}\text { E.D. } \\
\text { E. D. }\end{array}$ & $\begin{array}{l}\text { SV. A. } \\
\text { M. A. }\end{array}$ & $\begin{array}{l}\text { SV. A. } \\
\text { S. A. }\end{array}$ & S.D. & $\begin{aligned} \text { VD. } \text { E. D. } \\
\text { E.D. }\end{aligned}$ \\
\hline & 2 & E.D. & M. A. & \begin{tabular}{|l|} 
S. A. \\
S. \\
\end{tabular} & S. D. & E. D & SV. A. & M. A. & S. D. & E.D. \\
\hline & $\frac{3}{4}$ & $\begin{array}{l}\text { VDD\&\& D. } \\
\text { VD. \& D. }\end{array}$ & \begin{tabular}{|c|} 
M. A. \\
N.
\end{tabular} & \begin{tabular}{|l|} 
S.A. \\
N.
\end{tabular} & $\begin{array}{l}\text { S.D. } \\
\text { S. D. }\end{array}$ & $\begin{array}{l}\text { VD. \&D. } \\
\text { VD. \&D. }\end{array}$ & S. A. & S.A. & S.D. & $\begin{array}{l}\text { E.D. } \\
\text { S.A. \&. A. }\end{array}$ \\
\hline & 5 & S.t. & N. & N. & S. A. & S. A. \& A. & SV.A. & M. A. & M. A. & S.A. \& A A \\
\hline 2007 & $\begin{array}{l}6 \\
7\end{array}$ & $\begin{array}{l}\text { S.A. } \\
\text { S.A. } \\
\end{array}$ & \begin{tabular}{|l} 
M. A. \\
sv. A.
\end{tabular} & \begin{tabular}{|l|} 
S.A. \\
S.A.
\end{tabular} & $\left|\begin{array}{l}\text { M.A. } \\
\text { sv.A. }\end{array}\right|$ & $\begin{array}{l}\text { L. } \\
\text { S.A. }\end{array}$ & $\frac{\mathrm{N} \text { N. }}{\text { sV. A. }}$ & $\begin{array}{l}\text { EO. D. } \\
\text { M. A. }\end{array}$ & $\begin{array}{l}\text { S.A. A. } \\
\text { SV.A. }\end{array}$ & $\begin{array}{l}\text { S.A. \& A. A. } \\
\text { S.A. \& A. }\end{array}$ \\
\hline & 8 & S. A. \& A. & N. & EO. D. & M. A. & S. A. \&A. & N. & N. & sv. A. & S.A. \& A. \\
\hline & $\frac{9}{10}$ & $\begin{array}{l}\text { S.A. \& A. } \\
\text { S.A. \&.A. }\end{array}$ & $\frac{\text { SV. A. }}{\mathrm{N} .}$ & \begin{tabular}{|l|} 
M. A. \\
N.
\end{tabular} & M.A. & $\begin{array}{l}\text { S.A. \& A. } \\
\text { S.A. \& A. }\end{array}$ & $\begin{array}{l}\text { SV.A. } \\
\text { SV. A. }\end{array}$ & M.A. & $\mid \begin{array}{l}\text { M.A. A. } \\
\text { S.A. }\end{array}$ & $\begin{array}{l}\text { S. A. \&.A. } \\
\text { S.D. (D.) }\end{array}$ \\
\hline & 10 & $\begin{array}{l}\text { S.A. \& A. } \\
\text { E.D. }\end{array}$ & N. & \begin{tabular}{|l|} 
N. \\
EO. D.
\end{tabular} & \begin{tabular}{|l} 
S. A. \\
E. D.
\end{tabular} & $\begin{array}{l}\text { S. A. \& A. } \\
\text { E. D. }\end{array}$ & $\begin{array}{l}\text { SV. A. } \\
\text { N. }\end{array}$ & $\begin{array}{l}\text { S. A. } \\
\text { M. D. }\end{array}$ & $\mid \begin{array}{l}\text { S. A. } \\
\text { M. D. }\end{array}$ & $\begin{array}{l}\text { S. D. (D.) } \\
\text { E. D. }\end{array}$ \\
\hline & 12 & E. & N. & N. & M. D. & E. D. & N. & N. & M. D. & E. \\
\hline & $\frac{1}{2}$ & $\begin{array}{l}\text { E.D } \\
\text { E.D }\end{array}$ & \begin{tabular}{|l|} 
S. A. \\
M.A.
\end{tabular} & \begin{tabular}{|l|} 
S. A. \\
S.A.
\end{tabular} & $\begin{array}{l}\text { S. D. } \\
\text { S. D. }\end{array}$ & $\begin{array}{l}\text { E. D. } \\
\text { E. D. }\end{array}$ & SV. A. & $\begin{array}{l}\text { M. A. } \\
\text { S.A. }\end{array}$ & \begin{tabular}{|l|} 
S. D. \\
S.D.
\end{tabular} & $\begin{array}{l}\text { E. } \\
\text { E. }\end{array}$ \\
\hline & 3 & S. D. (D.) & N. & \begin{tabular}{|l|} 
S.A. \\
\end{tabular} & S. D. & S. A. \& A. & SV. A. & M. A. & S. A. & VD. \& D. \\
\hline & $\begin{array}{l}4 \\
5\end{array}$ & $\begin{array}{l}\text { S.A. A } \\
\text { S.D. (D }\end{array}$ & SV. A. & \begin{tabular}{|l|} 
VA. \\
N.
\end{tabular} & \begin{tabular}{|l|}
$\mid$ S. A. \\
S. A.
\end{tabular} & $\begin{array}{l}\text { S. A. \& A. } \\
\text { S.A. \& A . }\end{array}$ & $\begin{array}{l}\text { SV. A. } \\
\text { S. A. }\end{array}$ & $\begin{array}{l}\text { M. A. } \\
\text { S.A. }\end{array}$ & S. A. & S. D. (A.) \\
\hline 008 & 6 & S. A. \& & M. A. & \begin{tabular}{|l|} 
S. A. \\
\end{tabular} & M. A. & S. A. \& A. & SV. A. & M. A. & M. A. & $\begin{array}{l}\text { S.D. (A.) } \\
\text { S. A. A. }\end{array}$ \\
\hline & $\frac{7}{8}$ & S. A. \& A. & $N$. & \begin{tabular}{|l|} 
S. D. \\
S.
\end{tabular} & sv. A. & S. A. \& A. & $\begin{array}{l}\text { SV.A. } \\
\text { SV. }\end{array}$ & M. A. & $\begin{array}{l}\text { sv. A. } \\
\text { SV. }\end{array}$ & S.A. \&. A. \\
\hline & 8 & $\begin{array}{l}\text { S. A. \& A. } \\
\text { S. A. A. }\end{array}$ & & $\begin{array}{l}\text { SV.D. } \\
\text { V D. }\end{array}$ & $M$ & $\begin{array}{l}\text { S. A. \& A. } \\
\text { S. D. (D.) }\end{array}$ & S.A. & $\begin{array}{l}\text { S. A. } \\
\text { EO. D }\end{array}$ & s. & \\
\hline & 10 & L. & N. & N. & S. D. & S. D. (A.) & $\mathrm{N}$. & N. & S. A. & L. D \\
\hline & $\frac{11}{12}$ & E.D & N. A. & \begin{tabular}{|l|} 
S. D. \\
S.
\end{tabular} & $\begin{array}{l}\text { M.D. } \\
\text { S.D. }\end{array}$ & $\begin{array}{l}\text { E. D } \\
\text { E.D }\end{array}$ & N. & $\frac{N}{N .}$ & M. & E.D \\
\hline & 1 & & $\mathrm{~N}$. & SV.D. & VD. & & N. & S.D. & M. D. & E. \\
\hline & 2 & 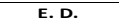 & $N$ & D. & & $E$ & N. & & $\overrightarrow{V t}$ & \\
\hline & 3 & E.D & $\mathrm{N}$ & M. D. & $\mathrm{M}$ & E. E & S. A. & S. A. & s. & \\
\hline & 4 & L. & $\mathrm{N}$ & N. & 3. & VD. \& D. & N. & N. & s. & D. \\
\hline & $\frac{5}{6}$ & L.D & N. & S.D. & S. D. & S. A. & SV.A. & M. A. & 0 & \\
\hline 2009 & $\frac{6}{7}$ & S.A. A. \& & $\frac{\text { SO.A. }}{\mathrm{N} .}$ & \begin{tabular}{|l|} 
M. A. A. \\
EO. D.
\end{tabular} & $\begin{array}{l}\text { M.A. } \\
\text { M. A. }\end{array}$ & $\begin{array}{l}\text { S.A. \& A. } \\
\text { S. A. \& A. }\end{array}$ & A. & S. A. & $\begin{array}{l}\text { M. A. } \\
\text { SV. A. }\end{array}$ & $\begin{array}{l}\text { S. A. \& A. A. } \\
\text { S. A. A. A. }\end{array}$ \\
\hline & 8 & $\begin{array}{l}\text { S.A. \& A. } \\
\text { S.A. \&.A. A }\end{array}$ & $\frac{\text { SV. A. }}{\mathrm{N}}$ & \begin{tabular}{|l|} 
S.A. \\
VD. \\
\end{tabular} & \begin{tabular}{|l|} 
M.A.A. \\
S.A.
\end{tabular} & $\begin{array}{l}\text { S.A. \& A. } \\
\text { S.A. \& A. }\end{array}$ & $\begin{array}{l}\text { SV.A. } \\
\text { SV. A. }\end{array}$ & S.A. & $\begin{array}{l}\text { M.A. } \\
\text { S.A. }\end{array}$ & $\begin{array}{l}\text { S. A. } \\
\text { S. A. }\end{array}$ \\
\hline & 10 & $\begin{array}{l}\text { S.A. \& } \\
\text { S. A. \& }\end{array}$ & $\frac{\text { N. }}{\text { SV. A. }}$ & \begin{tabular}{|l|} 
S. A. \\
S.
\end{tabular} & $\begin{array}{l}\text { S.A. } \\
\text { S. A. }\end{array}$ & $\begin{array}{l}\text { S.A. \&A. } \\
\text { S. A. \& A. }\end{array}$ & $\begin{array}{l}\text { SV.A. } \\
\text { SV.A. }\end{array}$ & M. A. & $\begin{array}{l}\text { 5. A. } \\
\text { S. A. }\end{array}$ & $\begin{array}{l}\text { S.A. \& A. } \\
\text { S. A. A. }\end{array}$ \\
\hline & 11 & E.D & $N$. & M. D. & M.D. & VD. \& D. & S. A. & N. & s. & E. \\
\hline & $\frac{12}{1}$ & $\frac{E}{E}$ & $\mathbf{N}$ & \begin{tabular}{|l} 
N. \\
sv.D.
\end{tabular} & \begin{tabular}{|l|} 
S.D. D. \\
M.D.
\end{tabular} & & $\frac{N}{N}$. & $\begin{array}{l}\text { N. } \\
\text { M. D. }\end{array}$ & $\begin{array}{l}\text { M. D. } \\
\text { M. D. }\end{array}$ & $\begin{array}{l}\text { E. } \\
\text { E. }\end{array}$ \\
\hline & 2 & $\mathrm{E}$. & $\mathbf{N}$ & N. & S. D. & E. & N. & N. & S.D. & E. \\
\hline & 3 & VD. \& & $N$ & $\mathrm{~N}$. & S. D. & VD. \& D. & N. & N. & S. D. & L. \\
\hline & $\frac{4}{5}$ & S. A & \begin{tabular}{|l} 
N. \\
sv. A.
\end{tabular} & \begin{tabular}{|l|} 
VD. A. \\
\end{tabular} & $\begin{array}{l}\text { S. D. } \\
\text { S. A. }\end{array}$ & & $\frac{\text { SV. A. }}{\text { N. }}$ & $\begin{array}{l}\text { M. A. } \\
\text { N. }\end{array}$ & S. A. & $\begin{array}{l}\text { VD. } \\
\text { S.A. }\end{array}$ \\
\hline 2010 & 6 & S. A. \& A. & N. & \begin{tabular}{|l|} 
V D. \\
\end{tabular} & S. A. & S. D. (A.) & N. & M. D. & S.A. & S. D. (A.) \\
\hline & $\frac{7}{8}$ & $\begin{array}{l}\text { S. A. \& A. } \\
\text { S. A. A. }\end{array}$ & $\frac{\text { SV. A. }}{\text { N. }}$ & $\frac{\text { N. }}{\text { N. }}$ & $\begin{array}{l}\text { SV. A. } \\
\text { sV. A. }\end{array}$ & $\begin{array}{l}\text { S. A. \&A. } \\
\text { S. A. \& A. }\end{array}$ & $\begin{array}{l}\text { SV. A. } \\
\text { SV. A. }\end{array}$ & $\begin{array}{l}\text { M. A. } \\
\text { S. A. }\end{array}$ & $\begin{array}{l}\text { SV. A. } \\
\text { SV. A. }\end{array}$ & $\begin{array}{l}\text { S. A. \& A. } \\
\text { S. A. A A. }\end{array}$ \\
\hline & 9 & S. A. \& A. & M. A. & $N$. & M.A. & S. A. \& A. & SV.A. & S. A. & V. A. & S.A.\&.A. \\
\hline & 11 & S.A. \&. & $\begin{array}{l}\text { N. } \\
\text { sv. A. }\end{array}$ & $\begin{array}{l}\text { EO. D. } \\
\text { M. A. }\end{array}$ & \begin{tabular}{|l|} 
M.D. \\
S. A.
\end{tabular} & $\begin{array}{l}\text { E.D. } \\
\text { S. A. \&A. }\end{array}$ & $\frac{\text { N. }}{\text { SV. A. }}$ & EA. D. & $\begin{array}{l}\text { M. D. } \\
\text { S. A. }\end{array}$ & ED. \\
\hline & 12 & E.t & N. & EO. D. & E.D. & E.D & N. & S.D. & M. D. & E.D \\
\hline
\end{tabular}


The stations whose names and features were set out in Table 7., their drought indices of AI, PNI, SPI, SPEI between 1881-2010 were calculated and the evaluation results were shown in in Table 8. -Table 13. Meanwhile, drought assessment was made on the basis of basin and the drought status of Konya Closed Basin is shown in Figure 2. As in Figure 2, when the aforementioned basin is examined for many years, the majority of the results for all indices occurred at normal or near normal levels, while extremely arid or extremely humid situations remained at very low rates. This situation demonstrated that both the index results were similar and the basin was not too dry between 19812010.

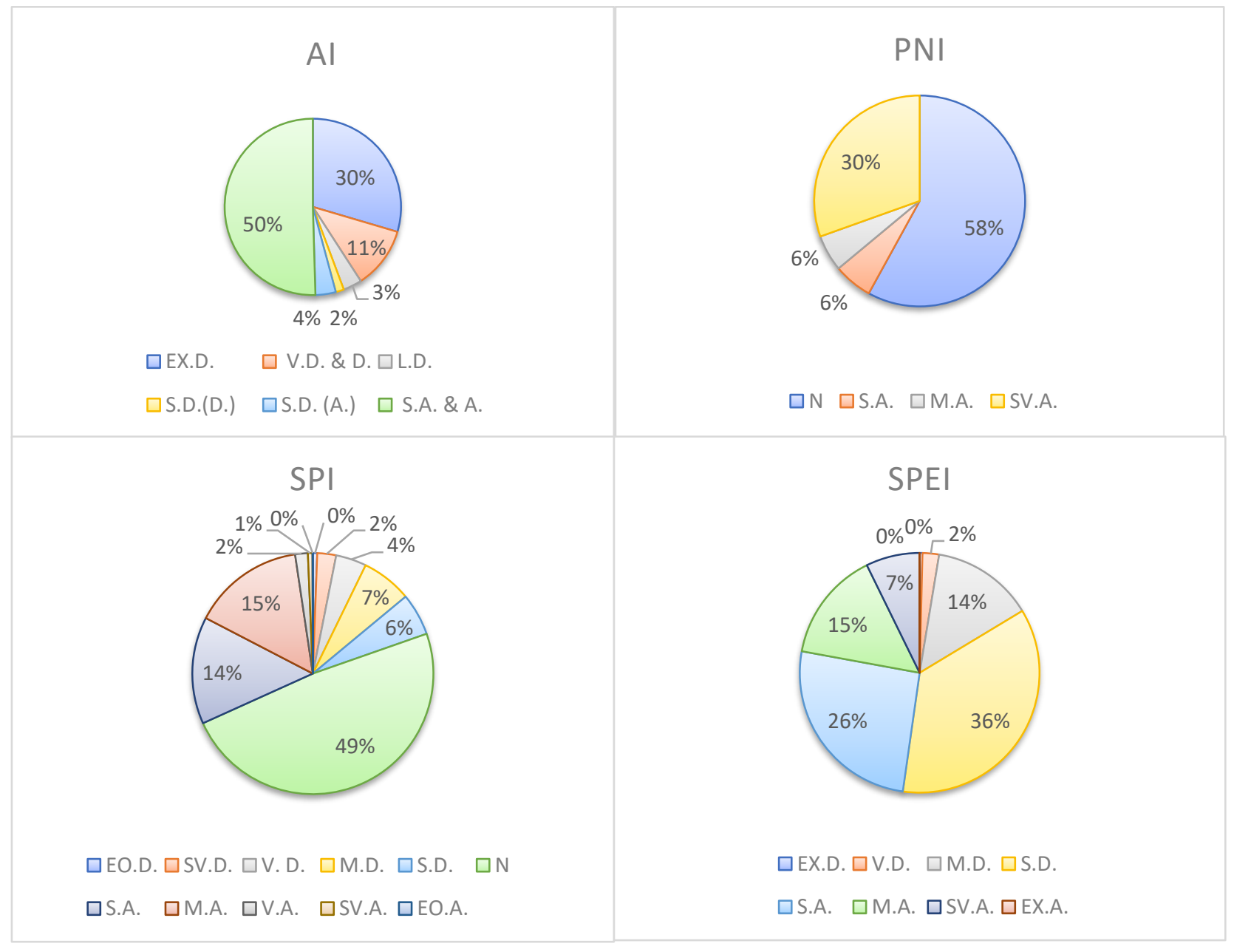

Figure 2. . Expression of AI, PNI, SPI and SPEI indices and drought calculation results in percentages. 
Descriptive statistics results of indices are given in Table 14.

Table 14. Descriptive Statistic Results of Indices

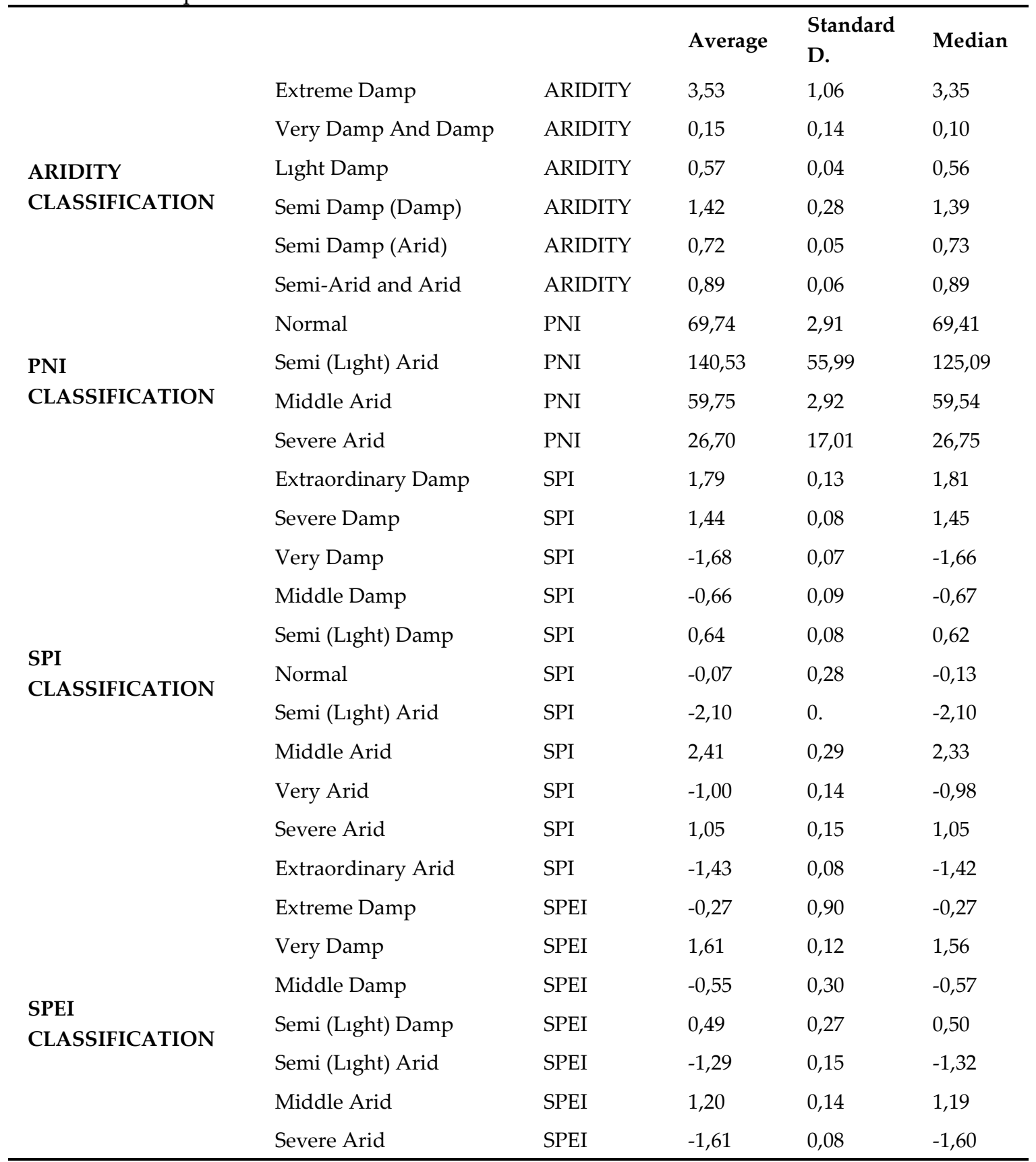

In this study, Exploratory Factor Analysis was conducted to test the computability of the indices used in the study as a single index, and Cronbach's Alpha coefficient were calculated in order to define reliability level of the test. Before the exploratory factor analysis, KMO and Bartlett tests were performed to understand suitability of the data for factor analysis. While the KMO coefficient was calculated to test the sample size, the normal distribution condition was examined with the Bartlett test. In factor analysis, factor load values were utilized in assigning scale items to factors or deleting from the scale. 


\begin{tabular}{lll}
\hline KMO & & \\
\hline & $X 2$ & 10381,781 \\
Bartlett Test & Sd & 6 \\
& $\mathrm{p}$ & 0,000 \\
\hline
\end{tabular}

Factor analysis results are given in Table 15 and $\mathrm{KMO}$ value was calculated as 0.528 in factor analysis. Accordingly, sample size was found to be suitable for factor analysis (KMO>0,500). Within the scope of Bartlett test, X2 value was calculated as 2220,024 and was found statistically significant $(p<0.05)$. Accordingly, the normal distribution condition has been met. Considering the results of $\mathrm{KMO}$ and Bartlett test, it is concluded that the data are suitable for factor analysis.

In order to calculate a single index from the indices, it was required to gather under one factor according to the principal components analysis, and as a result of the factor analysis, it was determined that the indices could be gathered under one factor. However, when the reliability coefficient was calculated, the value was determined as 0.058 . Therefore, by subtracting the PNI index from the factor, the Cronbach's Alpha coefficient increased from 0.320 to 0.711 is set out in Table 16 .

Table 16. Change of Cronbach Alpha value when indices are deleted

\begin{tabular}{ll}
\hline Index & $\begin{array}{l}\text { When the index is deleted } \\
\text { Cronbach's Alpha }\end{array}$ \\
\hline ARIDITY & 0,049 \\
PNI & 0,711 \\
SPI & 0,030 \\
SPEI & 0,052 \\
\hline
\end{tabular}

The PNI index was subtracted from the factor and the analysis was re-performed, and the results were found as in Table 17.

Table 17. Factor Analysis Results

\begin{tabular}{llll}
\hline Index & Factor Load & Variance Ratio & $\begin{array}{l}\text { Cronbach's } \\
\text { Alpha }\end{array}$ \\
\hline SPEI & 0,910 & & \\
ARIDITY & 0,906 & 65,330 & 0,711 \\
SPI & 0,557 & & \\
\hline
\end{tabular}

When the table was examined, it was determined that the SPEI, ARIDITY and SPI indices were gathered under a single factor. Accordingly, it was concluded that by taking the average of the indices, a general index can be calculated and used. As a result of factor analysis; a new index variable was found by taking the arithmetic mean of the SPEI, ARIDITY and SPI indices. K-Means Cluster Analysis was performed to determine the cutoff values of the new index and to create its categories. Each index is divided into 5 clusters (categories) with the method applied to determine the cutoff values of the categories with scientific methods. The cutoff values for the new index and the ANOVA test results are shown in Table 18.

Table 18. Cutoff Values and ANOVA Test Results for New Index Clusters 


\begin{tabular}{lllllll}
\hline & Ave. & Standard D. & Min. & Max. & F & p \\
\hline Cluster 1 & 1,46 & 0,23 & 1,11 & 1,88 & & \\
Cluster 2 & 0,71 & 0,20 & 0,39 & 1,10 & & \\
Cluster 3 & 0,05 & 0,19 & $-0,25$ & 0,39 & $\mathbf{1 1 8 6 0 , 3 3 6}$ & $\mathbf{0 , 0 0 0 ^ { * }}$ \\
Cluster 4 & $-0,55$ & 0,17 & $-0,98$ & $-0,25$ & & \\
Cluster 5 & 2,27 & 0,30 & 1,88 & 3,19 & & \\
\hline * & & & & &
\end{tabular}
${ }^{*} \mathrm{p}<0,05$

According to the result of cluster analysis with the new index, clusters are formed as follows.

- Cluster 4: values between -0.98 and -0.25

- Cluster 3: values between -0.25 and 0.39

- Cluster 2: values between 0.39 and 1.10

- $\quad$ Cluster 1: Values between 1.11 and 1.88

- $\quad$ Cluster 5: contains values between 1.88 and 3.19.

ANOVA test is performed to determine whether there is a difference between clusters in terms of index. According to ANOVA test result, the difference between new indices in different clusters is statistically significant $(\mathrm{F}=11860,336 ; \mathrm{p}<0.05)$. Accordingly, the cutting values formed in cluster analysis gave significant and effective results in dividing indices into different categories. The average lower and upper values of the new indices and clusters are shown in figure 3.

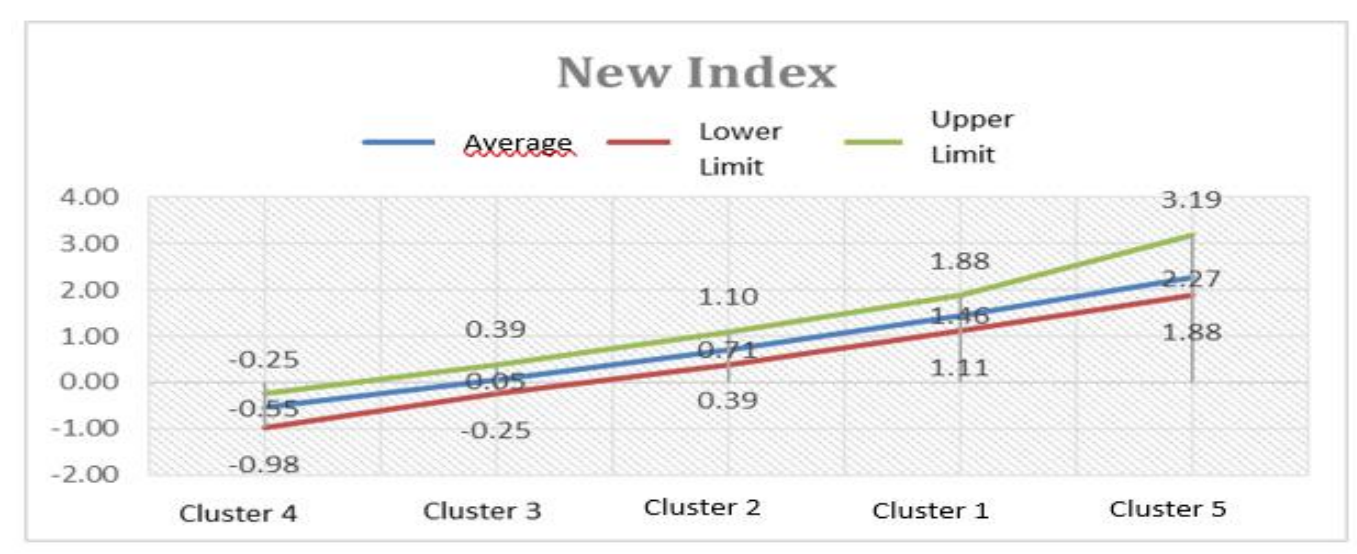

Figure 3. New index values

Table 19. Relationship between clusters in new index and ARDITE, SPI and SPEI categories 


\begin{tabular}{|c|c|c|c|c|c|c|c|}
\hline & & Cluster 1 & Cluster 2 & Cluster 3 & Cluster 4 & Cluster 5 & $\mathrm{p}$ \\
\hline & Extreme Damp & 196 & 115 & 1 & 0 & 141 & \multirow{6}{*}{$0,000^{*}$} \\
\hline & Very Damp and Damp & 0 & 29 & 431 & 1144 & 0 & \\
\hline ARIDITY & Light Damp & 0 & 16 & 137 & 0 & 0 & \\
\hline \multirow{8}{*}{ CLASSIFICATION } & Semi Damp (Damp) & 68 & 214 & 57 & 0 & 0 & \\
\hline & Semi Damp(Arid) & 1 & 37 & 77 & 0 & 0 & \\
\hline & Semi-Arid and Arid & 1 & 65 & 67 & 0 & 0 & \\
\hline & Extraordinary Damp & 22 & 19 & 6 & 0 & 20 & \multirow{11}{*}{$0,000^{*}$} \\
\hline & Severe Damp & 13 & 30 & 19 & 0 & 18 & \\
\hline & Very Damp & 0 & 0 & 3 & 10 & 0 & \\
\hline & Middle Damp & 25 & 51 & 106 & 335 & 3 & \\
\hline & Semi (Light) Damp & 22 & 47 & 71 & 18 & 8 & \\
\hline \multirow[t]{9}{*}{ SPI CLASSIFICATION } & Normal & 105 & 198 & 362 & 337 & 36 & \\
\hline & Semi (Light) Arid & 0 & 0 & 0 & 1 & 0 & \\
\hline & Middle Arid & 26 & 20 & 1 & 0 & 23 & \\
\hline & Very Arid & 9 & 53 & 108 & 377 & 0 & \\
\hline & Severe Arid & 42 & 53 & 75 & 0 & 33 & \\
\hline & Extraordinary Arid & 2 & 5 & 19 & 66 & 0 & \\
\hline & Extreme Damp & 28 & 55 & 79 & 116 & 12 & \multirow{7}{*}{$0,000^{*}$} \\
\hline & Very Damp & 0 & 0 & 0 & 0 & 21 & \\
\hline & Middle Damp & 2 & 50 & 350 & 387 & 0 & \\
\hline \multirow[t]{4}{*}{ SPEI CLASSIFICATION } & Semi (Light) Damp & 179 & 369 & 263 & 38 & 26 & \\
\hline & Semi (Light) Arid & 0 & 2 & 75 & 377 & 0 & \\
\hline & Middle Arid & 57 & 0 & 0 & 0 & 82 & \\
\hline & Severe Arid & 0 & 0 & 3 & 226 & 0 & \\
\hline
\end{tabular}

${ }^{*} \mathrm{p}<0,05$

The relationship of clusters formed as a result of clustering analysis with the new index with ARIDITY, SPI and SPEI categories were examined and presented in Table 19.

According to the analysis results; it has been observed from the new index that cluster 1 mostly matches the category of extremely humid, humid and very humid in ARIDITY, cluster 2 matches with extremely humid, humid and very humid, cluster 3 matches with arid and semi-arid and semihumid, cluster 4 matches with arid and semi-arid, cluster 5 matches with extremely humid. As stated by chi-square test results, the relationship between new clusters and ARIDITY category was found statistically significant ( $\mathrm{p}<0.05)$. It has been revealed from the new index that cluster 1 matches mostly in SPI with the normal and medium humid categories, cluster 2 matches with the normal, medium arid and medium humid categories, cluster 3 matches with the normal, medium arid and light arid categories, cluster 4 matches with medium arid, normal and light arid categories and cluster 5 matches with normal and medium humid categories. In accordance with the chi-square test results, the relationship between the new clusters and the SPI category was found statistically significant ( $p$ $<0.05)$. It has been observed from the new index that cluster 1 matches mostly in SPI with the light 
humid and medium humid categories, cluster 2 matches with light humid, extremely humid categories, cluster 3 matches with light humid, light arid categories, cluster 4 matches with the light arid and medium arid categories, and cluster 5 was found to match the medium humid and light humid categories. According to the chi-square test results, the relationship between new clusters and SPEI category was found to be statistically significant $(\mathrm{p}<0.05)$.

\section{Conclusions}

In the aforementioned study, drought analysis was carried out with the drought indices selected from the Konya Closed Basin sample and an assessment was made with the help of various statistical analyzes on which indices were stronger.

According to the evaluated analysis results; while the SPEI, SPI and ARIDITY indices were significant and strong, the contribution of the PNI index was found to be low in terms of significance. Our results demonstrated that the PNI index was not included in the indices which were recommended in drought analysis and gave significant guidance.

Also for the other three calculated indices (SPI, SPEI and ARIDITY); as a result of re-evaluation of the cutoff values of these indices and determination of new cutoff values, common use was tried to be examined. The results were set out in Table 17, Figure 3 and Table 18.

When the results obtained in the study are examined; by collecting different indices under one index, it was pointed out that it might be possible to make healthier analyzes on a micro and / or macro scale by recovering the problem of obtaining different results with more than one index by doing similar studies for many drought indices, on the basis of sub-basin, basin and country. 


\section{References}

1. Aghrab, A.; Bouabid, R.; Elalaoui, A. In Drought characterization using drought indices in two areas of the Mediterranean basin: Meknès, Morocco, and Córdoba, Spain

2. Sruthi, S.; Aslam, M. A. M., Agricultural Drought Analysis Using the NDVI and Land Surface Temperature Data; a Case Study of Raichur District. Aquatic Procedia 2015, 4, 1258-1264.

3. Leng, G.; Tang, Q.; Rayburg, S., Climate change impacts on meteorological, agricultural and hydrological droughts in China. Global and Planetary Change 2015, 126.

4. Lee, T.; Modarres, R.; Ouarda, T., Data-based analysis of bivariate copula tail dependence for drought duration and severity. HYDROLOGICAL PROCESSES 2013, 27, 1454-1463.

5. Zelenhasic, E., On the Extreme Streamflow Drought Analysis. Water Resources Management 2002, 16 (2), 105-132.

6. Raziei, T.; Bordi, I.; Pereira, L., An Application of GPCC and NCEP/NCAR Datasets for Drought Variability Analysis in Iran. Water Resources Management 2011, 25, 1075-1086.

7. Y.E, Y., Sulama Açısından Kuraklık Analizi. (Drought Analysis in Irrigation).)" Ege University Faculty of Agriculture Journal 2002, 39.

8. Akcay S., U. M. A. v. G. H., Aydın Yöresinde Sulama Yönünden Kuraklık Analizi. (Drought Analysis for Irrigation in Aydın Region). Ege University Faculty of Agriculture Journal 2007, 44 (1), 137147.

9. Hınıs M.A., Bütünleşik Kuraklık İndeksi ile Aksaray'da Hidrometeorolojik Kuraklık Analizi. (Hydrometeorological Drought Analysis in Aksaray with Integrated Drought Index). Journal of the Faculty of Engineering and Architecture of Gazi University 2013, 28, 711-721.

10. V, G.; M.S., Y.; O., S., Hidrolojik Kuraklık Değerlendirmesi: Murat Nehri-Palu Örneği.( Hydrological Drought Assessment: The Case of Murat River-Palu). Harran University Journal Of Engineering 2018, 3 (297-301).

11. Huang, Y. F.; Ang, J. T.; Tiong, Y. J.; Mirzaei, M.; Amin, M. Z. M., Drought Forecasting Using SPI and EDI under RCP-8.5 Climate Change Scenarios for Langat River Basin, Malaysia. Procedia Engineering 2016, 154, 710-717.

12. Danandeh Mehr, A.; Vaheddoost, B., Identification of the trends associated with the SPI and SPEI indices across Ankara, Turkey. Theoretical and Applied Climatology 2020, 139 (3), 1531-1542.

13. Akbari, M.; Asgari, H.; Sadoddin, A.; Khosravi, H., Drought Monitoring Based on the SPI and RDI Indices under Climate Change Scenarios (Case Study: Semi-Arid Areas of West Golestan Province). ECOPERSIA 2016, 4, 585-1602.

14. Mishra, A.; Singh, V., A Review of Drought Concepts. Journal of Hydrology 2010, 391, 202-216.

15. Labedzki, L., Estimation of local drought frequency in central Poland using the standardized precipitation index SPI. Irrigation and Drainage 2007, 56, 67-77.

16. Hayes, M. J.; Svoboda, M. D.; Wiihite, D. A.; Vanyarkho, O. V., Monitoring the 1996 Drought Using the Standardized Precipitation Index. Bulletin of the American Meteorological Society 1999, 80 (3), 429-438.

17. Tsakiris, G.; Tigkas, D.; Spiliotis, M., Assessment of interconnection between two adjacent watersheds using deterministic and fuzzy approaches. European Water 2006, 16.

18. Fiorillo, F.; Guadagno, F., Karst Spring Discharges Analysis in Relation to Drought Periods, Using the SPI. Water Resources Management 2010, 24, 1867-1884. 
19. Pamuk G., Ö. M. v. T. K., Standart Yağış İndisi (SPI) ile Ege Bölgesinde Kuraklık Analizi.( Drought Analysis in Aegean Region with Standard Precipitation Index (SPI)) Ege University Faculty of Agriculture Journal 2004, 41, 99-106.

20. Çelik M.A., K. İ. v. B. H., Doğu Anadolu Bölgesi'nin Mevsimlik Kuraklık Analizi.( Seasonal Drought Analysis of Eastern Anatolia Region.). Journal of Atatürk University Institute of Social Sciences 2018, 22, 1741-1761.

21. Ilgar, R., Çanakkale'de Kuraklık Durumu Ve Eğilimlerinin Standartlaştırılmış Yağış İndisi İle Belirlenmesi.( Determinatıon Of Drought Situatıon And Tendencies In Çanakkale With A Standardized Precipitation Indication). Journal Of Marmara Geography 2013, 0, 183-204.

22. Vicente-Serrano, S. M.; Beguería, S.; López-Moreno, J. I., A Multiscalar Drought Index Sensitive to Global Warming: The Standardized Precipitation Evapotranspiration Index. Journal of Climate 2010, 23 (7), 1696-1718.

23. Nastos, P. T.; Politi, N.; Kapsomenakis, J., Spatial and temporal variability of the Aridity Index in Greece. Atmospheric Research 2013, 119, 140-152.

24. Greve, P.; Roderick, M. L.; Ukkola, A. M.; Wada, Y., The aridity Index under global warming. Environmental Research Letters 2019, 14 (12), 124006.

25. Dave, V.; Pandya, M.; Ghosh, R., Identification of Desertification Hot Spot Using Aridity Index. Annals of arid zone 2019, 58, 39-44.

26. Nikbakht, J.; Tabari, H.; Talaee, P. H., Streamflow drought severity analysis by percent of normal index (PNI) in northwest Iran. Theoretical and Applied Climatology 2013, 112 (3), 565-573.

27. Li, T.; Li, S.; Liang, C.; Bush, R.; Xiong, L.; Jiang, Y., A comparative assessment of Australia's Lower Lakes water quality under extreme drought and post-drought conditions using multivariate statistical techniques. Journal of Cleaner Production 2018, 190.

28. Hasaninejad, A.; Taghdisi, A.; Noori, S. H.; Akbarian Ronizi, S. R., The Role Of Drought Risk Management In Reducing The Vulnerability Of Farmers: A Case Study Of The City Of Zarrindasht). Journal Of Rural Research 2018, 9 (2 \#P00293), -.

29. Pham, M.; Vanhaute, W.; Vandenberghe, S.; De Baets, B.; Verhoest, N., A copula-based assessment of Bartlett-Lewis type of rainfall models for preserving drought statistics. Hydrology and Earth System Sciences Discussions 2013, 10, 7469-7516.

30. Beharry, S. L.; Gabriels, D.; Lobo, D.; Clarke, R. M., A 35-year meteorological drought analysis in the Caribbean Region: case study of the small island state of Trinidad and Tobago. SN Applied Sciences 2019, 1 (10), 1256.

31. Motamed, M. K.; Devisti, H., Environmental and Socio-Economic impacts of Drought from the viewpoint of Guilan Paddy farmers, North of Iran. Caspian Journal of Environmental Sciences 2012, 10 (2), 227-235.

32. Éva, S.-A.; Péter, L.; Pauler, G.; Dénes, B.; Horvath, F., Drought Stress Distribution Responses of Continental Beech Forests at their Xeric Edge in Central Europe. Forests 2016, 7, 298.

33. Biamah, E.; Sterk, G.; Sharma, T. C., Analysis of agricultural drought in Iiuni, Eastern Kenya: Application of a Markov model. Hydrological Processes 19 (2005) 6 2005, 19.

34. Rahmat, S.; Jayasuriya, N.; Bhuiyan, M., Identification of homogeneous areas for drought frequency analysis. International Journal of Integrated Engineering 2017, 9 (2), 9.

35. Borgonovo, E.; Lu, X.; Plischke, E.; Rakovec, O.; Hill, M., Making the most out of a hydrological model data set: Sensitivity analyses to open the model black-box. Water Resources Research 2017. 
36. Thomas B. McKee, N. J. D., John Kleist, The Relationship Of Drought Frequency And Duration To Time Scales. In Conference on Applied Climatology, Anaheim, 1993; Vol. 8, pp 179-184.

37. Amini, A. A. K., A.; Al-Ansari, N.; Karami Moghadam, M.; Mohammad, T. Application of TRMM Precipitation Data to Evaluate Drought and Its Effects on Water Resources Instability. . Appl. Sci. 2019, , 9, 5377.

38. McKee, T. B.; N. J. Doesken; J. Kleist In Drought monitoring with multiple time scales, Ninth Conference on Applied Climatology, American Meteorological Society, , Dallas TX, Dallas TX, Jan1520, 1995; pp pp.233-236.

39. Willeke G., H. J. R. M., Wallis J.R. and Guttman N.B The National Drought Atlas; Institute for Water Resources Report: 1994.

40. Güner Bacanlý, Ü.; Akpan, G. N., Drought Analysis in Mediteranean Region. Pamukkale University Journal of Engineering Sciences 2019, 25 (6), 665-671.

41. Jamro, S. C., F.N.; Dars, G.H.; Ansari, K.; Krakauer, N.Y. , Exploring the Evolution of Drought Characteristics in Balochistan,Pakistan. Appl. Sci. 2020, 10, 913.

42. Jamro, S. D., G.H.; Ansari, K.; Krakauer, N.Y. , Spatio-Temporal Variability of Drought in Pakistan Using Standardized Precipitation Evapotranspiration Index. Appl. Sci. 2019,, 9, , 4588.

43. Türkeş, M. İklim Verileri Kullanılarak Türkiye'nin Çölleşme Haritası Dokümanı Hazırlanması Raporu. (Using Climate Data Desertification Map of Turkey Document Preparation Report) Ankara, 2013.

44. Stevens, J., Applied multivariate statistics for the social sciences. Mahwah, NJ: Lawrence Erlbaum Associates, 1996.

45. Bartlett, M. S., Tests Of Significance In Factor Analysis. British Journal of Statistical Psychology 1950, 3 (2), 77-85.

46. Kalaycı Ş., SPSS uygulamalı çok değişkenli istatistik teknikleri(SPSS applied multivariate statistical techniques.). Asil Publication Distribution, 2009; Vol. 4.

47. Nunnally, J. C., Psychometric Theory. 25 Years Ago and Now. Educational Researcher 1975, 4 (10), 7-21.

48. Ketchen, D. J.; Shook, C. L., The Application of Cluster Analysis in Strategic Management Research: An Analysis and Critique. Strategic Management Journal 1996, 17 (6), 441-458.

49. Hansen, P.; Jaumard, B., Cluster analysis and mathematical programming. Mathematical Programming 1997, 79 (1), 191-215.

50. Yaz, H. F. Çok Değişkenli İstatistiksel Yöntemlerden Kümeleme Analizi; SPSS ile bir uygulama (Cluster Analysis from Multivariate Statistical Methods; An application with SPSS). 2014.

51. Stoline, M. R., The Status of Multiple Comparisons: Simultaneous Estimation of all Pairwise Comparisons in One-Way ANOVA Designs. The American Statistician 1981, 35 (3), 134-141.

52. Larson, D. A., Analysis of Variance with Just Summary Statistics as Input. The American Statistician 1992, 46 (2), 151-152.

53. Büyüköztürk, Ş., Sosyal bilimler için veri analizi el kitabı (manual of data analysis for social sciences). Pegem Akademi Yayınları, 2017; Vol. 23.

54. Doğdu M.Ş., T. M. M., ve Sağnak C., , "Konya kapali havzasinda yağiş ve yeraltisuyu seviyelerinin irdelenmesi.( Investigation of precipitation and groundwater levels in Konya closed basin.). Turkey Climate Change Congress 2007.

55. Durduran, S. S., Coastline change assessment on water reservoirs located in the Konya Basin Area, Turkey, using multitemporal landsat imagery. Environmental Monitoring and Assessment 2010, 164 (1), 453-461. 
